\title{
Network science in clinical trials: A patient-centered approach
} \author{
Benjamin Haibe-Kains ${ }^{\mathrm{a}, \mathrm{b}, \mathrm{c}, \mathrm{e}, *}$ \\ a Bioinformatics and Computational Genomics Laboratory, Princess Margaret Cancer Center, Toronto, ON, Canada \\ ${ }^{\mathrm{b}}$ Department of Computer Science, University of Toronto, Toronto, ON, Canada \\ c Ontario Institute of Cancer Research, Toronto, ON, Canada \\ d Breast Cancer Translational Research Laboratory, Université Libre de Bruxelles, Brussels, Belgium \\ e Department of Medical Biophysics, University of Toronto, Toronto, ON, Canada \\ ${ }^{\mathrm{f}}$ Department of Pathology, GZA Hospitals Antwerp, Belgium \\ ${ }^{\mathrm{g}}$ Medical Oncology Clinic, Institut Jules Bordet - Université Libre de Bruxelles, Brussels, Belgium
}

Venkata S.K. Manem ${ }^{\mathrm{a}, \mathrm{e}, 1}$, Roberto Salgado ${ }^{\mathrm{d}, \mathrm{f}, 1}$, Philippe Aftimos $^{\mathrm{g}}$, Christos Sotiriou ${ }^{\mathrm{d}, \mathrm{g}}$,

\begin{abstract}
A B S T R A C T
There has been a paradigm shift in translational oncology with the advent of novel molecular diagnostic tools in the clinic. However, several challenges are associated with the integration of these sophisticated tools into clinical oncology and daily practice. High-throughput profiling at the DNA, RNA and protein levels (omics) generate a massive amount of data. The analysis and interpretation of these is non-trivial but will allow a more thorough understanding of cancer. Linear modelling of the data as it is often used today is likely to limit our understanding of cancer as a complex disease, and at times under-performs to capture a phenotype of interest. Network science and systems biology-based approaches, using machine learning and network science principles, that integrate multiple data sources, can uncover complex changes in a biological system. This approach will integrate a large number of potential biomarkers in preclinical studies to better inform therapeutic decisions and ultimately make substantial progress towards precision medicine. It will however require development of a new generation of clinical trials. Beyond discussing the challenges of high-throughput technologies, this review will develop a framework on how to implement a network science approach in new clinical trial designs in order to advance cancer care.
\end{abstract}

\section{Introduction}

In preclinical and clinical cancer research there is an increasing use of molecular throughput-technologies enabling us to leverage large quantities of molecular data. This is enhancing our knowledge of deregulated oncogenic pathways due to alterations at the genomic, transcriptomic and protein level $[1,2,3]$. This massive amount of data represents a challenge for the scientific community in terms of finding a relevant integrated approach capturing the dynamics and functional heterogeneity of metabolomics, transcriptomics and genomics $[4,5,6]$ in several different cellular compartments and locations of the same tumor and in the same patient.

The use of such networks and the development of gene-regulatory, protein-protein and human disease networks provide a promising tool to allow high-throughput network analysis [7]. A network science approach aims to capture the systemic nature of human disease as a whole, including the immune-system which represent an illustrative example where a network science approach can provide an added value $[8,9,10,11]$.

The use of network science is rarely used in drug development and not explored yet in the clinical trial setting. This may be related to knowledge gaps between clinicians, scientists and regulatory officials on this matter $[12,13]$. What can we learn from network science and how should this be applied in drug development and in a clinical trial setting? This review aims to illustrate the most important concepts of network science and discusses emerging biological concepts wherein network science approaches are essential for a thorough understanding of cancer biology and its applicability in a clinical-trial context. We will argue that a network science approach is to be considered as a next step facilitating the integration of genomics, transcriptomics, proteomics and other omics including morphology in drug development on the one hand and on the other hand into a patient-centered context in clinical trials.

\footnotetext{
* Corresponding author at: Bioinformatics and Computational Genomics Laboratory, Princess Margaret Cancer Center, Toronto, ON, Canada.

E-mail address: bhaibeka@uhnresearch.ca (B. Haibe-Kains).

${ }^{1}$ Equal Authorship.
} 
Undirected network
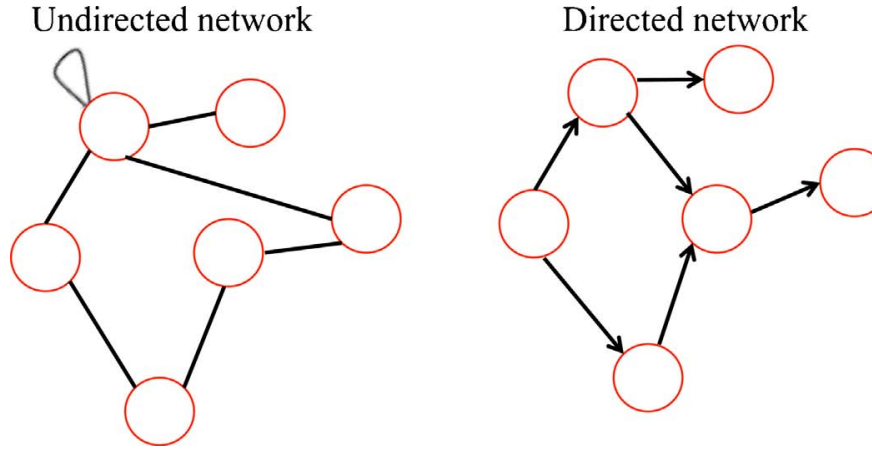

2. Network science and the non-linearity of cancer biology

\subsection{Non-linearity of tumor dynamics}

Cancer may be viewed as a complex and a heterogeneous disease resulting in uncontrolled growth of cells within the body. Some of the reasons include (but are not limited to) genetic changes, epigenetic alterations, micro-environmental variations, carcinogenic agents exposure and abnormalities in several key molecular functions in a cell $[14,15]$. Carcinogenesis is generally viewed as an evolutionary process, because the pre-malignant lesions have to undergo several mutations in order to become malignant cells $[16,17,18]$. The complexity and heterogeneity of carcinogenesis can be explained through somatic evolution where the process of cancer initiation and progression is governed through evolutionary mechanisms $[16,17,18]$. The advent of mutated clones and their fitness is controlled to some extent by its micro-environment, for example, the proliferative capacity (also, termed as the fitness) of a cell is a function of micro- and macro-environmental conditions in the tumor such as oxygen and metabolic nutrients in the system. In a study by Bozic et al. [19], the authors have shown that driver mutations confer a fitness advantage in glioblastoma and pancreatic cancers during tumor progression [19]. This drives the fact that tumor microenvironment confers a fitness advantage to tumor cells, eventually leading to invasion of neighboring tissue.

The technological improvements in sequencing technologies has enabled comprehensive characterization of the mutational landscape for various solid tumors including breast [20], brain [21]; [22], lung [23], pancreatic [24] and ovarian cancers [25,26].

In the multi-step process of carcinogenesis, many different types of mutations must occur before a cell turns malignant, and this has been already studied in the past $[27,28]$. This leads to the altered regulation of oncogenes and tumor-suppressor genes, eventually resulting in the dysfunction of different molecular pathways involving these affected genes [29]. These molecular pathways represent several biological processes relating to proliferation, cellular metabolism, immune signalling, epithelial-mesenchymal transition and many others. Several studies have supported the fact that tumor microenvironmental conditions, such as hypoxia, could potentially affect the gene regulation and alters the landscape of molecular pathways [30]. Furthermore, the spatial and temporal dynamics of these conditions determine the functional behaviour of the phenotype. For example, the glucose and oxygen concentrations in the tumor continuously vary over space and time [31], which has a detrimental effect on the metabolic pathways in the tumor. In order to understand how a malignant lesion is functionally different from a normal tissue over space and time, it is necessary to assess the complete landscape of signalling interactions rather than relying on the individual pathways.

Due to the fact that dynamics of cancer being highly nonlinear (defined as those changing conditions that do not occur in a linear way) in both space and time, one has to rely on network science and systems biology based approaches.
Fig 1. A schematic diagram of an undirected and a directed network.

\subsection{Network science and systems biology}

Systems biology is a research that focusses on the computational and mathematical methods which are supported by the underlying fundamental biological principles. While, network science is a field that focuses on the theoretical aspects of networks between biological entities, such as molecules, proteins, genes, etc. Using network science, we can model the cellular interactions across biological entities and apply systems biology techniques to address fundamental questions related to these interactions.

In the molecular biology landscape, complex intercellular and intracellular signalling interactions contribute to the functioning of several biological processes in a cell. This has facilitated the need to decode complex phenotypic traits in the tumor using biological networks, causing a paradigm shift in modelling these complex interactions [32]. The basic premise of these biological networks is that many components in the system interact with each other in a nonlinear and nonadditive manner. These networks can help us elucidate the spatial and temporal mechanisms behind the complex dynamics of biological systems. The availability of transcriptional data allows one to focus on the large-scale networks of interacting genes, rather than solely relying on individual genes or components of a system.

Fig. 1 displays a schematic diagram of an undirected network (where causal relationships/edge directions between nodes are ignored) and a directed network (where causality/edge directionality between nodes is accounted).

\subsubsection{Types of biological networks}

Biological networks can be broadly classified into two categories, namely, causal networks and association networks. The causal relationships between two random variables, or nodes can be captured using a causal interaction network. For example, a set of genes that influence a given gene's expression level can be represented using a causal interaction network. On the other hand, association networks are genetic networks that capture the association between any two entities such as proteins, or genes, or a disease-gene association. Below is a concise summary of different types of networks.

The relationship between various biological entities, for instance, molecules, genes, and proteins can be efficiently described as networks whose nodes represent these entities and edges denote the interactions between these entities. The link between genes and proteins form complex cellular networks, which include gene regulatory networks, protein-protein interaction networks, metabolic networks and genetic interaction networks. In gene regulatory networks, nodes are genes and transcription factors. edges between these nodes can be either directional or bi-directional, which in biological terms can be either inhibition or activation. Protein interaction networks are those networks which encode the information of proteins and their physical interactions. The signalling networks are those networks that capture the information between signalling proteins. These networks have revealed global topological properties that relate to known biological properties. Metabolic networks capture the biochemical reactions in the cell, where 
a)

\section{mRNA}

b)

\section{Proteins}

Metabolites

c)

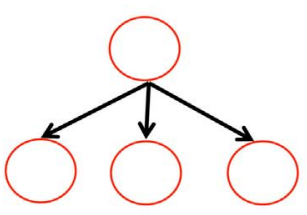

Gene Regulatory Network Node: Transcription factors Edge: Regulatory interaction
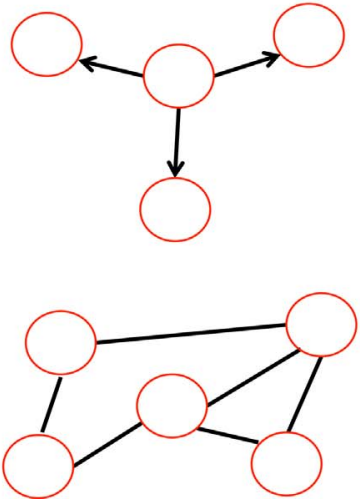

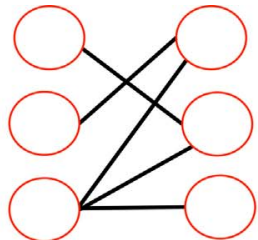

Co-expression Network Node: Genes

Edge: Co-expression relationship

(for example, correlation)

Protein-Protein Interaction Network

Node: Proteins

Edge: Interaction
Fig. 2. Schematic diagram of different types of networks: a) gene regulatory network, co-expression network; b) protein-protein interaction network; c) metabolic network.

\section{Metabolomic Network \\ Node: Metabolites \\ Edge: Reaction}

the nodes can be enzymes or substrates, and reactions can be directional or bi-directional. Edges in metabolic networks are closely related to functions of a gene, and has a great potential for applications in the biological interpretation of roles of specific genes. Genetic association networks captures the functional relationships between genes. For example, correlation based networks describe the correlation patterns among genes, i.e. the edges between two nodes represent how correlated is the gene expression.

Fig. 2 displays different types of networks, namely, gene regulatory network, genetic interaction network, protein-protein interaction network and metabolic network. In regulatory networks edges represent interactions between transcription factors and target genes. In genetic interaction networks such as correlation based networks, the edges denote the statistical correlation in expression patterns (taken after a threshold on the correlation based on False Discovery Rate). In proteinprotein interaction (PPI) networks, edges denote measured stable interactions between proteins. In metabolite networks, edges represent

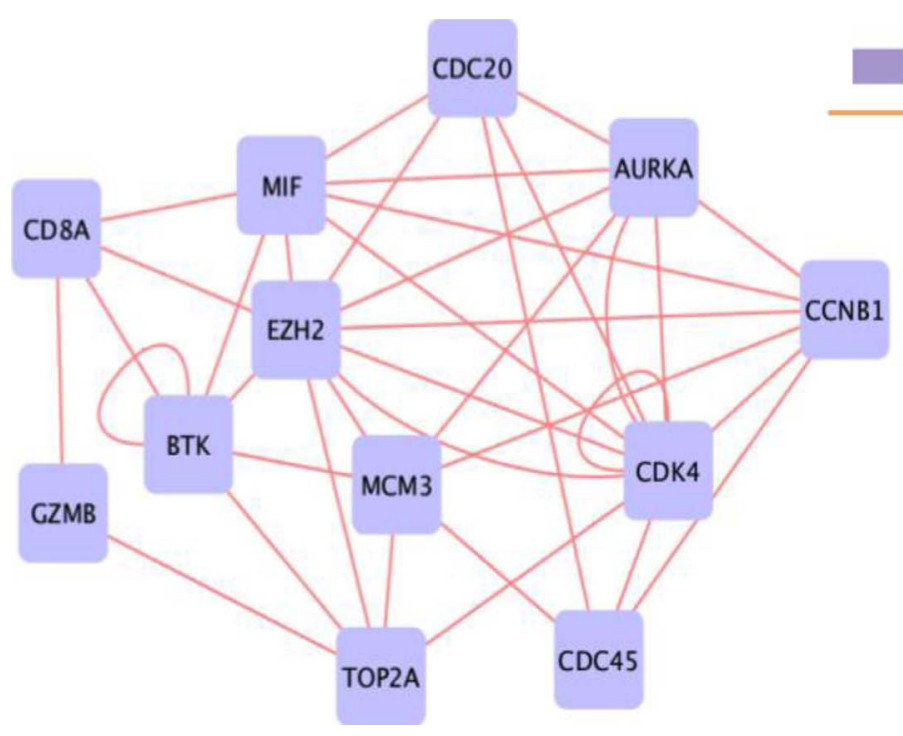

metabolic reactions and nodes denote compounds.

A sample biological network is illustrated in Fig. 3, which displays an undirected small genetic interaction network, a co-expression network. Each node in the network represent a gene, and the interactions in the network are the statistical correlations between the genes. In simple words, the edge quantifies the extent of similar pattern of gene expression between two conditions, or cellular phenotypes. This biological network is built using gene expression profiles derived from the laser capture microdissected transcriptional measurements of ER positive breast cancer patients.

\subsubsection{Network descriptors}

The topological and statistical properties of networks can provide valuable insight into the biological processes along with the internal organisational structure of interactions. These properties can be local (for instance, looking at a sub-graph) or global to a network. The identification of important nodes in the network is a non-trivial task,

\section{Genes}

Interactions

Fig. 3. Illustration of a gene-gene interaction sub-network. This biological network is constructed using gene expression profiles of ER + breast cancer patients. Each node denotes a gene and an edge represents the relationship between the nodes/genes. 
a)

b)

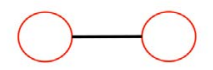

c)

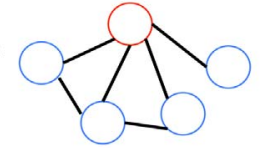

d)

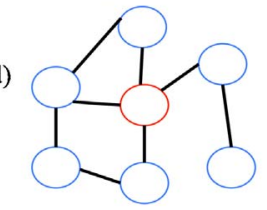

e)

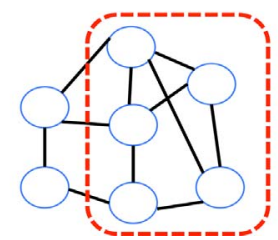

Node

Interaction relationship between two nodes

Degree: number of relationships connected to a node

Edge-betweenness: Number of times a given node appears in the shortest path between all other pairs of nodes

Community: Nodes that are densely connected within a group
Fig. 4. A schematic diagram of widely used network measures. a) Represents a node; b) two nodes with an interaction; c) degree of a node; d) edge-betweenness of a network; e) community or module of a network.

and there have been several research efforts dedicated towards this $[33,34,35,36]$. However, it is still a challenge to identify the nodes that drive a certain biological function, in a complex interaction network. Some of the widely known measures used to extract information from a network are degree, clustering, betweenness centrality and modules or communities. Fig. 4c-e displays well known network measures. The degree (defined as the number of connections) is used as a measure to indicate the importance of a node. For example, in Fig. 4c the degree of the red color node is four. Edge-betweenness is defined as the degree of centralization in a network, i.e. this measure describes the number of times a node appears in the shortest path of all pairs of nodes in the biological network. There are various betweenness centrality measures and a comparison of these can be found elsewhere [37].

Many clustering algorithms have been proposed in the literature to identify groups of nodes that participate in the same functional process [38]. For instance, clustering based approaches to PPI networks are broadly categorized into topology-free (which do not consider distance between proteins) and graph-based (considers topology of the network). However, the recent work by Padi et al. [39] suggests that a single network cannot capture the complete landscape of molecular interactions, and an appropriate network score is required that can be applied across different types of networks. Communities are defined as a group of nodes that have dense connections within a community and sparse connections across the communities. With regards to epi-stroma crosstalk, these communities represent groups of genes from epi and stroma that are tightly connected to each other. The interactions with these highly connected nodes in each community are tightly linked together and represent specific biological processes. Topologically, all the nodes within a community have more links to each other compared to other nodes in other modules.

Hubs in a biological network play a critical role in governing many biological processes and cellular functions. Although transcription factors are associated with high degree in the transcriptional network, however, they are not the biggest hubs in the biological network [39]. Also, in protein interaction networks, the hubs are the proteins which play a central role in several cellular processes [40,41]. Edge-betweenness measure has been applied on the protein interaction network to assess the protein druggability based on profiles of the druggable targets [42].

Although it is widely believed that disease related elements should be a part of hubs in the biological network [43], however, in a recent study by Platig et al., the authors demonstrated that disease associated SNP's (single nucleotide polymorphisms) are not global hubs within the network, but are local hubs pertaining to a network module [44]. These critical findings support the fact that non-hub genes indeed play a critical role in various biological functions. Therefore, the aforementioned measures underestimate the role of non-hub genes in the biological network [45]. Those nodes that can influence the flow of information in the network are termed as influential nodes, and these influential nodes can be hubs or non-hubs.

Over the last few years, identification of influential nodes has gained lots of interest and has numerous applications, for instance, in disease control and in social networks. In a recent study, investigators identified influential nodes which are part of the wound healing process using transcriptomic profiling data [46], and Gibbs et al. in another study captured all the influential nodes in the yeast gene regulatory network [47]. Recently, Morone and Makse [48] proposed an algorithm that efficiently computes the optimal influencers that dismantles the network into smaller components and disrupts the spread of information. Although this concept has been successfully applied in the context of spread of infectious diseases, there has been a void in its application to the cancer research.

\section{Network science and the tumor microenvironment}

\subsection{Tumor heterogeneity}

Cancer is a system of heterogeneous population of clones along with the complex web of network interactions among these clones, including with surrounding normal cells, which is termed as the intra-tumor heterogeneity (ITH) [49]. This complexity is one of the primary hurdles in understanding the evolutionary dynamics of cancer [50,51].

The phenotypic variations along with the tumor microenvironment (TME) dictate the changes in the gene expression. The TME is composed of non-cancerous cells present in the tumor, which include fibroblasts, immune cells and endothelial cells that form the blood vessels. The current targeted treatment paradigm is developed to target certain molecular aberrations based on the diagnosis carried out on the bulk tumor. Therefore, it is not surprising to observe intrinsic and extrinsic drug resistance in patients [52,53]. One better way to investigate ITH is through single cell sequencing, which is discussed in the next section. Understanding the ITH will facilitate effective therapies that could potentially target the driving forces of the ITH. Gerlinger et al. constructed a phylogenetic tree and showed that renal carcinoma has a branched evolutionary growth. The authors in their study also conveyed that bulk tumor sequencing from a single biopsy leads to an under estimation of mutational burden, due to ITH [54]. Benerji et al. explored the signalling pathways using network entropy and observed de-differentiation of cells and demonstrated the applicability of cancer evolution in a clinical setting [55]. Later, Park et al. used entropy based measure at the gene level to quantify the ITH on the TCGA pan cancer cohort [56]. To capture the complete evolutionary landscape of ITH, it is important to build biological networks based on data obtained from the temporal and spatial sequencing of the tumor.

\subsubsection{Tumor stroma}

A growing body of literature has shed light into the functional and phenotypical diversity during the carcinogenesis. The tissue homeostasis is perturbed by the mutated epithelial cells and change the surrounding microenvironment [57], termed as reactive stroma. The reactive stroma is characterized by a change in collagen bundles, degradation of basement membrane and ECM remodelling [58]. These include recruitment and differentiation of immune and endothelial cells as well as cancer-associated fibroblasts (CAFs), which together enhance tumor growth and metastasis. This in turn promotes aberrant cellular behaviour and the mutated cells co-evolve along with the stromal environment [58]. The communication between the tumor epithelial and 
stromal cells is regulated by a dynamic network of growth factors, cytokines and chemokines. The complexity of these inter and intracellular communications are mediated through regulatory loops between the tumor epithelial and stromal compartments. Variations in the tumor microenvironment (TME), in particular, the tumor-associated stroma, are linked to worse clinical outcome in aggressive cancer types, such as triple negative breast, colon, prostate, and ovarian cancers. Recent biological and clinical studies have recognized that tumor TME is a key contributor to cancer progression, invasion, metastasis and resistance to therapies $[59,60,61]$.

The variations in microenvironmental conditions result in the rewiring of molecular interactions between and within biological compartments. These rewired changes can yield novel biological insights into gain or loss of interactions specific to a condition. On this front, a recent study used a systems biology approach and analyzed epithelialstromal co-expression relationships between normal and tumor samples in breast cancer patients [62]. The authors observed the functional rewiring of self-loops (defined as those genes that are coexpressed in both the tumor epithelium and stroma) as an important feature of breast tumorigenesis. Identification of the epi-stroma crosstalk signature could enable us to find drugs that inhibit the epi-stroma co-dependencies.

\subsubsection{Immunological tumor context}

It is apparent that the immune-system is important in the development and progression of cancer [63] and that the assessment of immunological biomarkers at the morphological level -tumor infiltrating lymphocytes- as well as at the cellular level are important both for prognosis as well as for prediction in a variety of tumor-types $[64,11,65]$.

Similar approaches have been used for the analysis of adaptive immune-response in response to vaccination for example [66,67] enabling the analysis of individual cellular and subcellular interactions of immune-responses [68]. Chaussabel and colleagues propose an analytical strategy based on modular transcriptional repertoires of immunecell constituents relying on a priori determined co-dependent gene sets. This modelling avoids the classical reductionistic approaches like hierarchical clustering and principal component analysis, that are based on similarities of gene expression rather than based on biological dependencies of the variables assessed [68]. In this respect, Tan and colleagues reconstructed T-cell activation networks using phosphoproteomic profiling integrating multi-omics technologies and computational pipelines elucidating the temporal mechanisms of exit from T-cell quiescence. This multilevel approach allowed for better sensitivity compared to single-level omics since missing data in one approach can then be complemented by other measurements, illustrating another specific characteristic of multi-level network science approaches [69].

Within the context of simulating the determinants of response to immunotherapeutics, d'Onofrio and colleagues have modelled that the response rate on immunotherapeutics and the ability of periodically given treatments to remove of tumors depended on the aggressiveness of the tumor [70,71]. The biggest caveat however of those in silico modelling approaches is that there is still uncertainty whether this mere in silico modelling captures the dynamics of the interactome between tumor and immune cells. An alternative approach has been developed by Spitzer and colleagues using mass cytometry data of single immune cells from different organs enabling the development of immune-reference maps. This was used to map the immune organisation across different body compartments, taking into account the different genetic composition of the immune cells [10]. Using this approach an organism-wide study was conducted in genetically engineered cancer models. In this study it became evident that a coordinated systemic immune-response was necessary for optimal local response to immunotherapeutic agents, identifying through this network-approach that CD4-T cells primed at the periphery are important to initiate tumor response. These findings illustrate that a network science approach is applicable beyond the mere local micro-environmental setting [9].

The emergence of resistance to immunotherapeutic treatments needs to take into account the dynamic and continuously evolving nature of the interactions of the immune-system with not only the tumor-cells but also with the stromal cells [72,73]. This is illustrated in a patient with widely metastatic melanoma that had heterogeneous response to anti-PD1 therapy with some lesions regressing and some being resistant. Whole exome sequencing, gene expression assays and immunohistochemistry for PDL1 and CD8, among others, were applied to a set of 26 specimens ( 4 pre-mortem and 22 post-mortem). It became evident that there was a remarkable similarity at the genetic and immunological level between regressing and progressing cutaneous metastases, whereas gene expression profiles were able to distinguish a differential adaptive response between progressive versus responding lesions [74].

There is also evidence that the dynamics of the neoantigen landscape of anti-PD1 treatment-resistant NSCLC change after an initial response, wherein loss of 7-18 mutation-associated neoantigen clones was associated with the resistant samples through the elimination of tumor subclones, altered levels of T-cell receptors or importantly through deletion of chromosomal regions containing truncal alterations [75].

Furthermore, alterations at the chromosome level combined with tumor mutational load have been demonstrated to be better predictors of survival after immunotherapy than either alone [76]. The dynamic nature of the immune-infiltrate is also illustrated by the fact that the spatio-temporal dynamics of 28 different immune cells, called as "the immunome" infiltrating colorectal cancer changed at tumor-progression and these dynamics influences survival [77]. Immune-related gene expression signatures discriminates patients with and without metastases demonstrating also that immuno-cytotoxicity decreases with tumor-progression whereas other features such as driver mutations and chromosome instability were not related to the occurrence of metastasis [78].

Recently, a "cancer immunogram" was proposed that includes the following variables: (1) Tumor Foreignness "mutational load"; (2) General Immune-status "Lymphocyte count"; (3) Immune cell infiltration "Intratumoral T-cells"; (4) Absence of checkpoints "PDL1"; (5) Absence of soluble inhibitors "IL6, CRP"; (6) Absence of inhibitory tumor metabolism"LDH, glucose utilisation" and (7) Tumor sensitivity to immune effectors" MHC-expression, IFN-gamma sensitivity" [79] and references herein. The authors conclude their proposal stating that "this information required from this analysis may be obtained from the combination of tumor genomics, immunohistochemistry, and standard assays on the peripheral blood compartment".

A network science approach may thus be a critical and necessary tool to have an integrated analysis of all these variables.

\subsection{High-throughput spatial OMICS}

Exploring the phenotypic identity in a tumor requires the gene expression profiles at multiple locations in the tumor of individual phenotypes. The following sections present an integration of network science approaches from data derived from the high-throughput sequencing of spatial composition of tumor phenotypes along with single-cell sequencing.

\subsubsection{Spatial transcriptomics}

The clinical management of patients diagnosed with cancer remains dependent on a diligent evaluation of biopsies by pathologists. For instance, in breast cancer, pathological evaluation is usually supplemented by validated assays for ER, PR, and HER2 status. Network science approaches have been developed in estrogen-receptor positive disease finding correlations between expression modules and grade and tumor size respectively, as well as with survival, identifying hub-genes in this setting $[80,81]$. 
However, there is a pressing need to develop assays based on morphological and functional characteristics of various phenotypes in the bulk tumor. It is important to deconvolute the bulk tumor signal to various phenotypic components. In this regard, spatial genomics-transcriptomics plays a critical role in dissecting the bulk tumor to various phenotypes such as percentage of (reactive) stroma, tumor cells, lymphocytes, etc. The mapping of spatial heterogeneity of the tumor microenvironment using digital pathology tools is emerging as an important tool linking the morphological heterogeneity with high throughput technologies. Using spatial genomics-transcriptomics it is possible to delineate the molecular surrogates of the spatial heterogeneous localisation of lymphocytes or proliferating tumor cells [82].

Multiplexed profiling-technologies of immune-subtypes are currently being developed that may potentially be used in a clinical trial setting, herewith providing a future rationale to couple spatial genomics to high throughput-technologies and network science-approaches. This concept will also promote further understanding of the microenvironmental heterogeneity and integration of genomic information herein. This concept can be further elaborated on by using a spatial transcriptomics approach on tissue sections whereby a co-localisation of proteins, morphological features and RNA-profiling in situ will enable us to identify and map the spatial heterogeneity of not only the tumor cells, but as well as the spatial features of the microenvironment [83] in which the immune-component is gaining tremendous importance in this era of immunotherapeutics. Mapping the spatial features of a tumor is becoming important since drug-penetration in the stroma and consequent development of resistance is equally influenced and determined by the heterogeneity of the microenvironment [84,85]. Integrating spatial genomics-transcriptomics into a network-science approach may lead to a more unbiased approach of the patients tumor compared to a less integrated approach wherein this variable is not taken into account.

\subsubsection{Single-cell sequencing}

Single-cell RNA sequencing is an emerging technique that can be used to assess cell-to-cell variability at the genomic scale. Unlike bulk tumor sequencing, single cell RNA sequencing provides resolution of transcriptomic details to the level of individual cells, for example, in a highly heterogeneous cancer such as triple-negative breast cancer. Single cell sequencing technology offers the possibility of identifying tumor subpopulations and selecting appropriate combinations of drugs that could potentially target each individual subpopulation, thus decreasing chances of tumor resistance.

Lee and colleagues performed single cell RNA sequencing on 10 breast cancer cells which were treated with paclitaxel. They found distinctly different gene expression profiles between stressed cells and drug-tolerant cells [86]. They additionally studied clonal evolution of a single drug-tolerant cell by sequencing each of the cells in its sixth generation. In another study, hundreds of differentially expressed genes were found between just 8 sequenced cells of melanoma tumor cells using a custom single cell RNA sequencing protocol [87].

Recently, Crow et al. constructed single cell co-expression network from 31 individual studies comprising 163 individual cell types, and investigated the diversity in the functional characteristics of cells [88]. The authors compared networks made from distinct populations of specific cell types to networks containing all of the cell types assayed within an experiment. In this way, they assessed the effects of cell-state and compositional variation on functional connectivity. In their methodology, networks were built using Spearman correlation, and each edge weight was defined as the rank of the correlation coefficient within the network. The degree of a node was calculated as the summation of all the weights connected to a given node. Aggregation was performed by averaging ranked correlation coefficients across networks, then re-ranking and standardizing values between 0 and 1 [88].

In another study, Wang et al. did a comparative study between single cell and bulk tumor gene expression profiles. They found distinct features for the co-expressed genes between single cell and bulk tumor data.

Applying network principles on single cell data could potentially help us to explore the biological complexity and functional heterogeneity within the bulk tumor at a much higher resolution.

\section{Identification of drug targets using network science}

\subsection{Network pharmacology}

Several statistical approaches have been used to characterize the biological mechanisms of molecular interactions, however, networks have pioneered in describing, identifying and characterizing the regulatory mechanisms involved in cellular networks. By associating the topological features of networks under differentially expressed conditions, one can identify putative genes that regulate the transcriptional program. Another important goal in cancer research is stratification of heterogeneous population of patients in a biologically and clinically meaningful manner. Creixell et al. [89] provided an overview of network-based pathway analysis, which is used to infer the putative gene interactions that vary across multiple conditions, or in normal versus malignant states.

The traditional approach of drug design and discovery relied on "one target-one drug". For many years, this approach has influenced identification of actionable targets, classification of disease, drug screening as well as in the design of clinical trials.

Hopkins described networks as a promising tool for drug development [90]. This allowed a paradigm shift from the single-target to a multi-target approach, with a network centric view of drug-target interactions. In this regard, systems biology has laid down the foundation to enhance knowledge of multiple targets in cancer at multi-pathway level through networks.

Networks can be used to identify multiple nodes (either genes or proteins) that can inhibit or activates a pathway to a given compound. This ideal methodology can be used to identify pathways leading to the drug resistance, and help find another synergistic compound that can act in coherence with the other drug. Recent years had seen the application of systems biology based approaches in drug design and discovery $[91,92,93,94]$. Jaegar et al. used network based approach and discovered novel synergistic drug combinations in breast cancer using the concept of pathway crosstalk inhibition [95]. Halasz and colleagues describe a two-stage computational model that enables the construction of signal transduction networks, and used to predict the therapeutic effectiveness of agents that targets the network in different cells or tissues [96]. Network models can also be used to predict the bypass mechanisms of cancer cells when a critical "node" is targeted, or knocked down form the system. Astsaturov et al. [97] built an EGFR (epidermal growth factor receptor) centered protein network, and identified subnetworks of proteins influencing resistance.

\subsection{Identification of signalling alterations}

Cancer cells have the characteristic of constantly being rewired at the genetic and epigenetic level [98]. "Rewired" in this context means that, tumor cells are constantly undergoing evolutionary changes at the genetic and epigenetic level, which is reflected in the inferred networks representing the various associations between the biological entities. This has as a consequence therapeutic failure as emerging resistant clones emerge over time due to changing environmental conditions and natural selection, with several different mutational spectra and subtypes being identified [99,100,101,102,103].

How these mutation spectra and mutational alterations affect the dynamics of signalling networks is of importance for adequate drug development. It is in this regard that the concept of cancer network attractors can be introduced, which is defined by the perturbation of signalling pathways rendering, changing or "attracting" the malignant phenotype [104]. Computational strategies have been developed 
$[105,106]$ to analyse the interaction of somatic alterations with the signalling processes within the same tumor. Performing a pan-cancer analysis on 3281 samples from 12 different cancer types have elaborated up to 16 significantly mutated subnetworks exhibiting co-occurring mutations across different samples.

Of interest, it has been shown that gene expression features are more informative than mutations for prediction of response to targeted therapies [107]. The interaction between tissue-, each with its unique microenvironment and the genomic context will determine whether a patient will respond or not to a particular treatment, i.e., having the same genetic alteration in different tumor types may not elicit the same response [108]. Therefore, models that incorporate causality between mutational findings with transcriptomics on the one hand and proteomics on the other hand are urgently needed [5]. This has particular importance for finding druggable targets in tumor types that have rare and low frequency mutated genes, wherein the use of other non-network science may probably be suboptimal for this purpose.

Creixell and colleagues have named signalling altering mutations as network attacking mutations (NAMs) [109]. Using a set of ovarian cancer cell lines, cancer genome repositories and a computational platform called ReKINect they have defined three classes of NAMs: (1) NAMs that disrupt signalling network dynamics by constitutively activating a protein kinase, thereby maintaining the information flow "on" or "off" uninterrupted over time; (2) NAMs that rewire upstream or downstream interactions, either of the mutated protein or node, and, (3) NAMs that destroy or generate phosphorylation sites, generating new molecular gates in cells. Conceptually they correlate with node inactivation/activation as loss-of-function mutations and gain-of-function mutations, illustrating the translation of classical mutation terminology into node-network science type of terminology.

Using this approach they have identified previously unknown phosphorylation sites supporting the hypothesis that cancer cells can acquire novel signalling flows leading to new phenotypic states. This approach illustrates an important caveat in current research, namely the genomic interpretation at the signalling level of the network-attacking mutations.

In summary, network biology is a useful tool in identification of actionable targets and at the same time could potentially decrease the failure rates in the drug discovery.

\section{Network science approach from the bench to the clinic}

\subsection{Current paradigms for precision-medicine studies}

Randomized clinical trials (RCTs) have been for decades the gold standard for clinical research and represent the backbone of regulatory approvals for novel therapies. Limitations of RCTs such as study duration, large sample size, statistical challenges in specific subgroups and cost have contributed to the emergence of adaptive designs [110]. Four case studies are illustrated in the review by Bhatt et al: seamless phase 2-3 design, sample size reestimation, changing the primary endpoint, and biomarker-driven adaptive population-enrichment designs.

The latter design applies to current-day oncology after the emergence of high-throughput DNA sequencing technologies that led to the molecular characterization of most histological cancer types. Indeed, highly active targeted agents in a setting of oncogene addiction have resulted in some cases in regulatory approvals following the results of phase 1 clinical trials [111] while other target-drug combinations still needed evidence from phase 3 RCTs before regulatory approvals $[112,113]$. These somatic activating alterations in oncogene addiction settings remain however rare, and represent a major obstacle when designing pivotal clinical trials. Indeed, the example of the neurotrophic tyrosine receptor kinases NTRK1, NTRK2 and NTRK3 fusions are prevalent mechanisms of oncogenic activation of this receptor tyrosine kinase family [114] while therapies in this setting yield encouraging response rates [115]. Thus the need for transformative clinical trial designs. Two notable examples are the histology-based clinical trial design to evaluate multiple molecular biomarkers also known as the "umbrella" trial; and the histology-independent, aberration-specific clinical trial also known as the "basket" trial [116].

"Umbrella" trials serve to tackle the paradigm of common cancers becoming rare and notable examples are the BATTLE study in NSCLC [117] and SAFIR-01 in breast cancer [118]. This was made possible since the mutational landscapes of the most prevalent cancer types have been deciphered and new molecular classifications have been identified $[119,120,121,122,123,124]$, allowing potential stratification to treatments with multiple drugs in one clinical trial enrolling the patients with one histological type. Patients with a detected genomic alteration are randomized to a standard therapy, chemotherapy in most cases, versus the genomically-informed targeted agent. An ongoing example is the lung-MAP trial in the second line treatment of squamous cell lung cancer with multiple randomized sub-studies within the principal studies (http://www.lung-map.org). Other trials such as SAFIR-02 in breast cancer studies the precision medicine paradigm by randomizing patients to 2 arms, a standard maintenance therapy arm versus maintenance therapy using a genomically-informed targeted agent (NCT02299999). "Umbrella" trials match patients to targeted agents based on single genomic alterations (hotspot mutations, copy-number variations, rearrangements, gene-expression profiles). A few trials also include potential immune response predictors such as mismatch repair deficiency immunohistochemistry (http://www.focus4trial.org/ aboutfocus4/focus4trialschema).

At the other end of the spectrum, the same genomic alterations can occur in a variety of cancer types [125]. These genomic alterations are however rare by cancer type, leading to the design of "basket" trials testing one biological agent targeting the same molecular alteration in different tumor types. One example of a "basket" trial in orphan diseases is the EORTC-CREATE trial testing crizotinib (MET and ALK inhibitor) in tumor types as rare as anaplastic large cell lymphoma, inflammatory myofibroblastic tumor, papillary renal cell carcinoma type I, alveolar soft part sarcoma, clear cell sarcoma and alveolar rhabdomyosarcoma (NCT01524926). Many such trials have already been reported such as PARP inhibitors in patients with germline BRCA1 or BRCA2 mutations with different tumor types [126] and BRAF inhibitors in tumors harboring the BRAF V600 hotspot mutation [127].

Such studies have yielded so far mitigated results because the patient and tumor contexts mattered beyond the detection of a potential oncogenic alteration. Indeed, feedback activation of EGFR was identified as a mechanism of resistance to BRAF inhibitor monotherapy in colorectal cancer harboring BRAF V600E mutations [128], explaining the absence of efficacy of single agent BRAF inhibitors in this setting. Other innate or acquired mechanisms of resistance could explain resistance to single-agent BRAF inhibitors in other tumor types. Vemurafenib was therefore combined with cetuximab, a monoclonal antibody targeting EGFR, in the colorectal cancer cohort of the vemurafenib "basket" trial [127]. The current development of "basket" trials supports the concept of agnostic clinical trial design [129], based on the targeting of one single genomic biomarker.

\subsection{Limitations with the current approaches}

The example of BRAF mutations in colorectal cancer highlights the complexity of the "contexts" in the prediction of drug efficacy and a non-exhaustive list of contextual variables include: co-occurring somatic genomic alterations, germline mutations harbored by the patient (both disease-predisposing and pharmacogenomic), immune cell infiltration and the stroma, epigenetics, expression and proteomics, sanctuary sites of metastases.

An adaptive clinical trial design using systems to transversally incorporate contextual components could help overcome the "reductionist paradigm" [130]. Indeed, multiple feedbacks and crosstalks between signalling pathways can mediate therapeutic escape and the 
interaction of drug targets with other protein can determine a discrepancy in tumor response in different tumor types. However, despite many examples of successful adaptive trials in cancer medicine such as I-SPY2 for example [131,132], the same reductionist approach continues to be applied. The reductionist approach is also challenged by heterogeneity and branched evolution [54]. Sequential strategies including re-challenges [133] and new generation of targeted agents [134] are used to overcome acquired resistance mechanisms and could be incorporated longitudinally in an adaptive clinical trial design based on systems biology. A recent example demonstrates the potential of integrating parallel (phospho)proteomic and mRNA sequencing data to interpret the context-specific impact of somatic alterations in PIK3CAmutant cell lines in terms of functional signatures such as (phospho) protein and transcription factor activities, supporting the incorporation of multiple functional readouts in a systematic stratification of tumors beyond single driver mutations [129,105].

The massive generation of data provided by next-generation sequencing, currently integrated in publicly available databases [135], could serve to infer functional genomic approaches that will be integrated in a computational framework applied to select candidate targets for biomarker-driven trials. The candidate targets would then be studied in cell lines and animal models, leading to the design of a therapeutic hypothesis to be tested in a clinical trial. The clinical development of Seribantumab, an ERBB3-targeting agent, followed this path for the bench to the clinic [136]. Preclinical prediction of sensitive phenotypes using systems biology would serve for patient selection in histology-based (umbrella) and aberration-based (basket) biomarkerdriven clinical trials, both at enrollment and dynamically with sequential testing.

\subsection{Complementary approach using network science}

\subsubsection{Pre-clinical studies supporting a network science approach}

The importance of having an overall context of the disease for patient management, beyond the mere importance of genomic drivers is illustrated by the finding of non-mutational drivers of resistance [137], the finding that synonymous mutations frequently act as driver mutations in human cancer [138] and that pseudogenes may contribute together with more classical genomic drivers to the transcriptional landscape of cells, its cellular differentiation and cancer progression [139]. Smith et al. demonstrate the non-oncogenic protein MITF-dependent drug-tolerance, wherein inhibiting MITF sensitizes the tumor cells again to BRAF and NRAS-inhibition.

The synonymous mutations, defined as changing the nucleotide sequence but not the sequence of the protein, contained within mostly oncogenes and not tumor suppressor genes, are not only tissue-dependent, but also alter exonic motifs that regulate splicing. Also, pseudogenes are identified in several tumor types and lineages increasing proliferation and migration in in vitro assays. There is also an emerging importance of the non-coding cancer genome wherein alterations in non-coding enhancers affect gene-expression in several tumor types [140]. Furthermore, the Cancer Genome Atlas Research Network performed an extensive molecular profiling of lung adenocarcinoma using mRNA, microRNA, DNA-sequencing integrated with copy number, methylation and proteomic analysis. Of interest, MAPK and PI3Kpathway activation could only be attributed to known mutations in a fraction of cases, suggesting that additional pathways of activation, despite the tremendous effort done so far, still need to be found [141].

The integration of genomic tools and integration with clinical variables was also assessed in a study by Yuan and colleagues [142]. Herein it was evident that the clinical variables as well as the genomic variables had limited added value when combined together, expect for a limited number of tumor-types, illustrating the redundancy of clinically useful information that genomics and clinics provide.

All the above illustrates that although a reductionistic linear approach has had some success benefiting patients, the limits of it may soon be reached.

A network-based approach may thus be able to capture the information of the information-system of the patient's tumor better and in a more complete manner than either approach alone. Adequately validated network science assay will also be able to incorporate different types of molecular and morphological data into a patient's individual network, optimizing the use of current high throughput technologies like whole genome sequencing, RNA-sequencing, etc, and morphological features like amount of tumor infiltrating lymphocytes whereas today these are rarely included in genomic clinical trial designs. Combining data from different sources has been exemplified in a study of prostate cancer combining a set of 232 SNP's, 6 plasma protein biomarkers and 5 standard clinical variables, demonstrating improved performance compared with PSA alone for the detection of tumors with a Gleason-score of at least 7 [143], illustrating that an integrated approach may improve the assessed variable (screening, prediction, prognosis, etc.) compared with a reductionistic approach.

\subsubsection{A patient-centered approach}

A major advantage of network-based approaches is that they encourage patient-centered approaches. Pemovska and colleagues have developed an Individual Systems Strategy based on a systematic ex vivo drug sensitivity and resistance testing and extensive molecular profiling of the malignant cells in patients with Acute Myeloid Leukemia (AML) [144]. A complementary method would be to use a network science approach not only for drug discovery by itself but also for elucidating whether combinational administration of cancer drugs is superior to a combinational approach. Lee and colleagues have demonstrated, using high density time-dependent measurements of signalling networks, gene expression profiles and cell phenotypic responses in combination with mathematical modelling, that the identification of rewired apoptotic pathways involving caspase-8 may render tumor cells more chemosensitive with sequential treatment having more effect than a combinational treatment [145].

The importance of elucidating the mechanisms of resistance through data-driven network modelling has been exemplified in an additional study demonstrating that in melanoma cells NRAS has both growth promoting and survival properties, whereafter inhibition of NRAS with MAP-ERK inhibition only blocked the survival property, but not the proliferative property, explaining the appearance of resistance to this particular treatment. In this reasoning, combining anti-survival with anti-proliferative drugs may thus be beneficial [146].

These examples are illustrative of the added value of using datadriven modelling approaches, reviewed in [147]. As such we conclude that reductionist approaches may be complemented by network biology.

\subsubsection{An example of potential clinical application}

The treatment of HER2-positive breast cancer is one setting that can exemplify the concepts developed above. This might appear counterintuitive as drug development in this setting has been very successful both for metastatic [148] and early disease [149,150,151]. Metastatic and relapsing patients are however not cured and new drug development in this setting continued successfully based on the presence of one sole predictive biomarker, HER2 overexpression/amplification, with the regulatory approvals of lapatinib [152], pertuzumab [153] and TDM1 [154].

Ongoing phase 3 clinical trials are continuing on the same model both in the adjuvant setting with pertuzumab (NCT02132949) and the metastatic setting with neratinib (NCT01808573). However, molecularly characterized HER2-positive breast cancer is not homogeneous and one example is the difference in response to neoadjuvant chemotherapy combined with anti-HER2 agents between ER + /HER2+ early breast cancer and ER-/HER2 + early breast cancer with a significantly higher rate of pathological complete response in ER-/HER2 + tumors. Furthermore, a subgroup of patients with HER2 + early breast 
cancer achieve a pathological response with chemotherapy-free double HER2-blockade, hinting towards a difference in biology [155,156].

Indeed, whole-genome sequencing (WGS) as well as gene-expression profiling (GEP) followed by intrinsic subtype classification were performed on a cohort of 64 HER2 + breast tumors and HER2 amplification was considered by the authors to be a genomic alteration common to a group of tumors in the basal to luminal spectrum and not as a determinant of a unique subtype of breast cancer [157]. The difference in response to anti-HER2 agents based on co-occurring somatic genomic alterations has been described in biomarker analyses of the pivotal phase 3 clinical trials proving than more than a single biomarker might be necessary [158]. On the other hand, data on the predictive and/or prognostic value of germline cancer-predisposing mutations is scarce or nonexistent in the setting of HER2+ breast cancer. Indeed, $3 \%$ of patients with BRCA1 or BRCA2 germline mutations develop HER2 + breast cancer [159]. These patients have been so far excluded for clinical trials testing PARP inhibitors. Beyond BRCA, other germline cancer-predisposing mutations are associated with HER2 + breast cancer such as TP53 mutations $[160,161]$ and warrant investigation as contextual variables to guide treatments for HER2 + breast cancer. While some anti-HER2 agents target the extracellular domain of HER2 and small molecules target the intracellular tyrosine kinase domain, transcription and expression of the genome data play a predictive role for treatment efficacy and emerging techniques are quantification of HER2 mRNA [162] and HER2 protein [163].

The integration of metabolic imaging such as zirconium-89-trastuzumab labelling has served as a prospective method for mapping the heterogeneous expression of the target and predicting response to HER2-targeting agents [164].

Finally, in this era of immunotherapy revival, the stroma and tumorinfiltrating lymphocytes (TILs) are being extensively studied and the correlation between lymphocytic infiltration and recurrence has been demonstrated in the adjuvant setting [165]. In the metastatic setting, TIL infiltration was found to be correlated to improved survival in the CLEOPATRA trial [166], providing the rationale for therapies that boost the patient's immune system. Synergism between anti-HER2 agents and immune checkpoint inhibitors has been demonstrated preclinically [167] and clinical trials are currently ongoing with the combination of trastuzumab and pembrolizumab (NCT02129556) and the combination of T-DM1 and atezolizumab (NCT02924883).

\subsection{Clinical network assay recommendations}

The use of a network-science approach may be particularly useful to capture the linear and nonlinear aspects of cancer biology, and be as such relevant for drug development and the development of predictive assays. If these types of approaches are introduced into the clinics for treating patients, a rigorous analytical and clinical validity followed by a trial design aimed at determining clinical utility is imperative.

The Institute of Medicine recently provided recommendations ranging from clinical utility to regulatory issues [168] on OMICS based approaches. A clinical trial protocol need not be static and should be adaptable to be able to incorporate new emerging knowledge. It is evident that the development of a complex signature, in this context thus a signature derived from a network-based assay including genomic and non-genomic sources need to follow the same rigorous process before considering its use in a clinical trial setting aimed at influencing the treatment of patients with cancer. Lisa McShane and colleagues have developed a list of 30 criteria for omics-based assays ranging from sample handling and requisites hereof, performance evaluation of the assay, the clinical trial designs ending with regulatory and legal criteria.

All these elements should be addressed to determine the applicability of any omics-based assay [169]. It may be assumed that a network-based assays are similar in nature to an omics-based approach, thus it is evident that the criteria developed by McShane and colleagues apply in a similar manner to network science approaches. In a complementary approach, FDA has recently issued a perspective on the regulatory implications of complex signatures to predict response to targeted therapies, ranging from regulations applicable to in vitro diagnostic devices to companion tests [170].

Challenging aspects of assay performance validation include analytical issues like cut-off issues, precision, reproducibility and replicability, robustness, limit of detection, etc. The development of a network-based assay for clinical use raises tremendous challenges and if not addressed adequately with statistically powered designs and patient sizes, rigorous analytical and clinical validity, the classification of the patient may be dubious $[171,172]$.

Michiels and colleagues have recently proposed a set of evidencebased criteria for prognostic signatures from laboratory to clinical practice, ranging from proof of concept, followed by analytical and clinical validity, with subsequent demonstration that the signature adds information to existing clinico-pathological information, has impact on clinical decision making and is cost-effective at the end [173].

Integrating networks in the design of adaptive trials hold the promise of linking drug activity to a baseline cancer context and could guide the design of combinatorial strategies to overcome both intrinsic and acquired drug resistance. Table 1 presents the advantages of using an network based approach compared to an OMICS based method in a clinical trial setting.

In the next section we propose a preclinical trial concept with a patient-centered clinical trial model including spatial genomics with immunogenomics, single or bulk molecular profiling, clonal and morphological heterogeneity. Considering the novelty and unprecedented nature of this approach, we follow the recommendations of Mogil and MacLeod to balance the demand for scientific novelty with the need for rigorous quality while preparing for a seamless transition between

Table 1

Differences between OMICS based and Network Science based approaches to clinical trial design.

\begin{tabular}{|c|c|c|}
\hline OMICS based trials & Network based trials & Comments \\
\hline Individual genes, a.k.a. gene signature [131]; [132] & Interactions between genes & $\begin{array}{l}\text { Networks can encompass all the possible interactions of predictive } \\
\text { features, providing more insight into patient-specific biology [174]; } \\
\text { [175] }\end{array}$ \\
\hline $\begin{array}{l}\text { Prediction of drug responsiveness (NCT02132949, } \\
\text { NCT01808573) }\end{array}$ & $\begin{array}{l}\text { The complex biological relationships } \\
\text { induced by the drug can be captured } \\
\text { through networks }\end{array}$ & $\begin{array}{l}\text { OMICS based models assume that genes are independent entities and } \\
\text { respond to treatments independently. But, drugs will also affect other } \\
\text { genes and pathways, which could potentially play a role on defining } \\
\text { the responsiveness of a drug to a patient [90] }\end{array}$ \\
\hline Complex interpretation of the disease etiology [130] & $\begin{array}{l}\text { Facilitate interpretation of the disease } \\
\text { etiology }\end{array}$ & $\begin{array}{l}\text { Multivariate models are complex to interpret compared to network } \\
\text { based methods. The process of inference of a disease and related it to } \\
\text { the value of the response variable is non trivial [176]. Interactions } \\
\text { among variables are easy to interpret in networks }\end{array}$ \\
\hline $\begin{array}{l}\text { Integration of heterogeneous data types is challenging and } \\
\text { prone to possible inconsistent conclusions due to noise } \\
\text { in each data type [130] }\end{array}$ & $\begin{array}{l}\text { Networks are more robust to noise and } \\
\text { data type heterogeneity }\end{array}$ & $\begin{array}{l}\text { Similarity Fusion Network (SNF) is a powerful network based } \\
\text { approach that integrates various data types representing a complete } \\
\text { landscape of the disease [177] }\end{array}$ \\
\hline
\end{tabular}




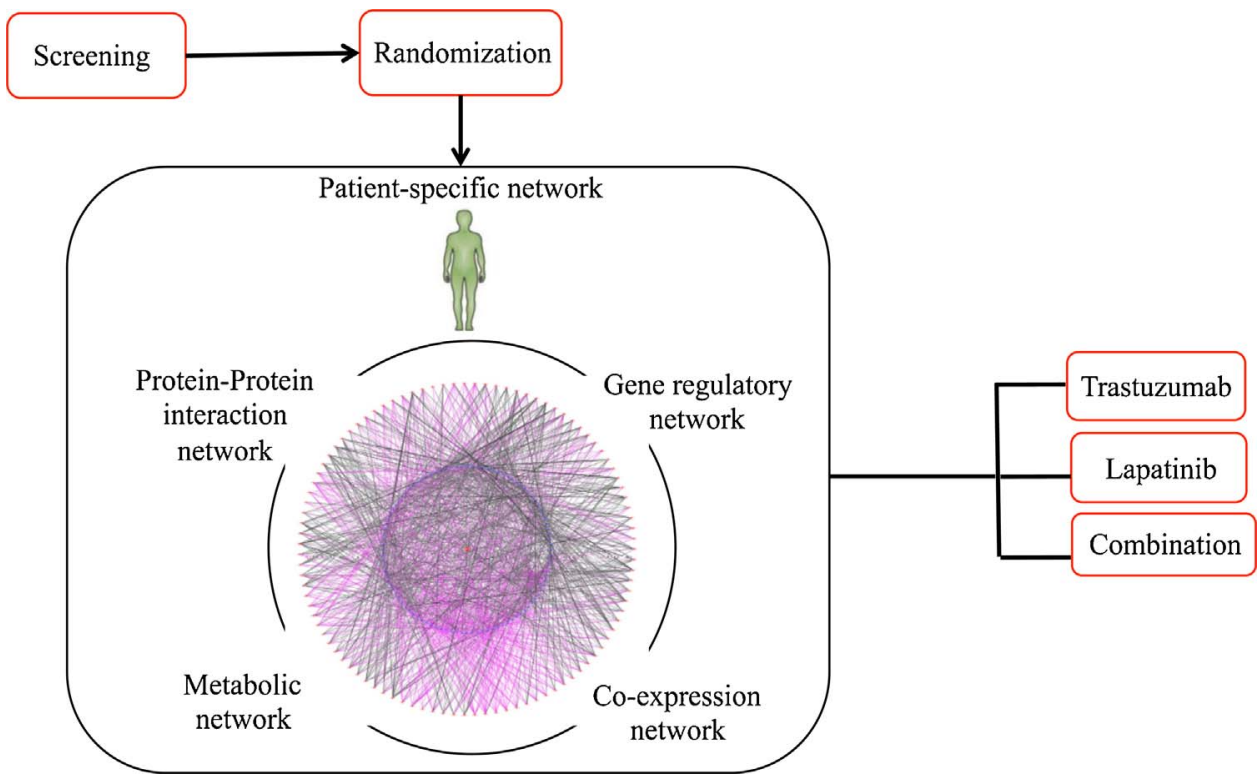

Fig. 5. An example of a clinical trial design where patients are randomly assigned to one of the three treatment arms, namely, treatment with Trastuzumab, Lapatinib, and a combination treatment with both the drugs. This randomization of patients can be replaced with network science approaches by constructing patient specific networks. preclinical research and clinical trial implementation. However, our main goal remains to test the innovative approach presented here [178].

\section{An example of integration of networks in clinical trial design}

A schematic diagram of a randomized clinical trial design implemented with three treatment regimens, namely, treatment with trastuzumab, lapatinib, and a combination treatment with both the drugs is displayed in Fig. 5. We assume here that molecular profiling data of all those patients who have responded and non-responded to trastuzumab, lapatinib, and a combination of both are available from earlier studies.

In general, a network construction method utilizes all the samples from a cohort and builds the network, termed as the aggregate network. In simple words, the aggregate network that represents the cohort can be viewed as a linear combination of networks from individual samples in the cohort. The following network methodology deconvolutes the aggregate network (constructed using $\mathrm{N}$ samples) of a given population into a group of individual, patient-specific networks. In particular, this method addresses the problem associated with phenotypic heterogeneity in the tumor samples in the cohort, and could potentially help the oncologists stratify patients according to their tumor biology.

There are two key aspects to our proposed methodology in a clinical trial setting: (i) construction of patient-specific network; (ii) treatment selection based on the patient-specific networks. These patient-specific networks can be explored retrospêctively to refine our biological understanding of the tumor response in the context of gene interactions. Kuijjer et al. recently introduced LIONESS (Linear Interpolation to Obtain Network Estimates for Single Samples), a network construction framework to infer patient-specific networks based on the transcriptomic profiling of the tumors. This method provides an opportunity to investigate and explore therapeutic opportunities for individual patients [179].

1. An aggregate network is constructed using all the samples from a given set of patients (Fig. 6). Correlation and mutual informationbased measures can be used to construct the aggregate network, say with the mRNA expression profiles using all the samples in the cohort.

2. We then extract individual patient networks that contribute to the aggregate network. LIONESS framework assumes that an aggregate network that is constructed without the patient of interest represents a common network [179]. The difference in this individual network with the aggregate network represents a perturbation to that network, effectively modelling the gene interactions specific to the patient tumor, presented in Fig. 7.

3. Clustering is a method of grouping samples into clusters such that those within each cluster are closely related to each other than those in other cluster. Once we obtain patient-specific networks from step 2 , we perform a hierarchical clustering on each patient specific network. This helps us identify homogeneous groups of patients who are clustered together. This framework could potentially stratify the patients and assign them to a treatment regimen. Another main advantage of performing the hierarchical clustering is that one can obtain outliers (if any) within the cohort. One of the important aspects of clustering is the distance metric that will be used to cluster patient-specific networks as a group.

4. There are several algorithms that are proposed in the literature to compare networks [180,181,182,183,184]. Also, several modes of network comparison has been discussed in [185]. These network comparison algorithms are promising tools to apply in this context and group a homogeneous set of patients to any treatment regimen. Comparison of two or more interaction networks can address the following key questions: a) which gene-gene interactions and groups of interactions are likely to have equivalent functions across a group of patients, for example in responders? b) Based on these similarities, can we obtain novel insights into the functional information about these putative gene-gene interactions that are poorly characterized in non-responders? c) What do these (groups of) interactions convey us about the biology of the (outlier) patient?

In Fig. 8, Groups 1, 2 denote patients treated with Trastuzumab, Lapatinib. Clustering of all patients will result in two clusters, i.e. all patients treated with Trastuzumab will fall in one cluster and all patients treated with Lapatinib will be in another cluster. If a patient does not cluster with any group, then that patient is called as an "outlier" and further investigation needs to be carried out on that patient. This could convey the following to the oncologist: a) the patient needs more genomic tests to investigate the biological or functional information which is different from other patients; b) needs a different treatment regimen, for example, a sequential treatment protocol. To summarize, this methodology can also be used to assign a patient to a given treatment arm and at the same time identification of patients who might not respond to a given treatment regimen (even before administration of the drug). 


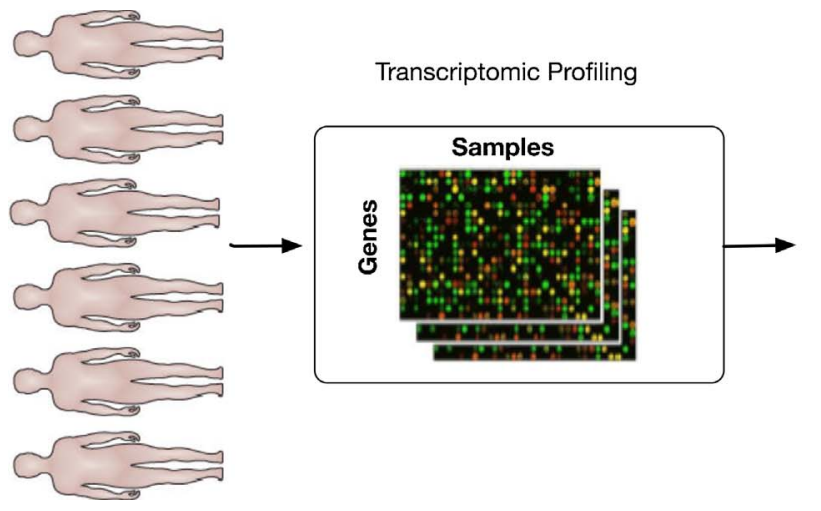

Fig. 6. Construction of aggregate network using all samples.

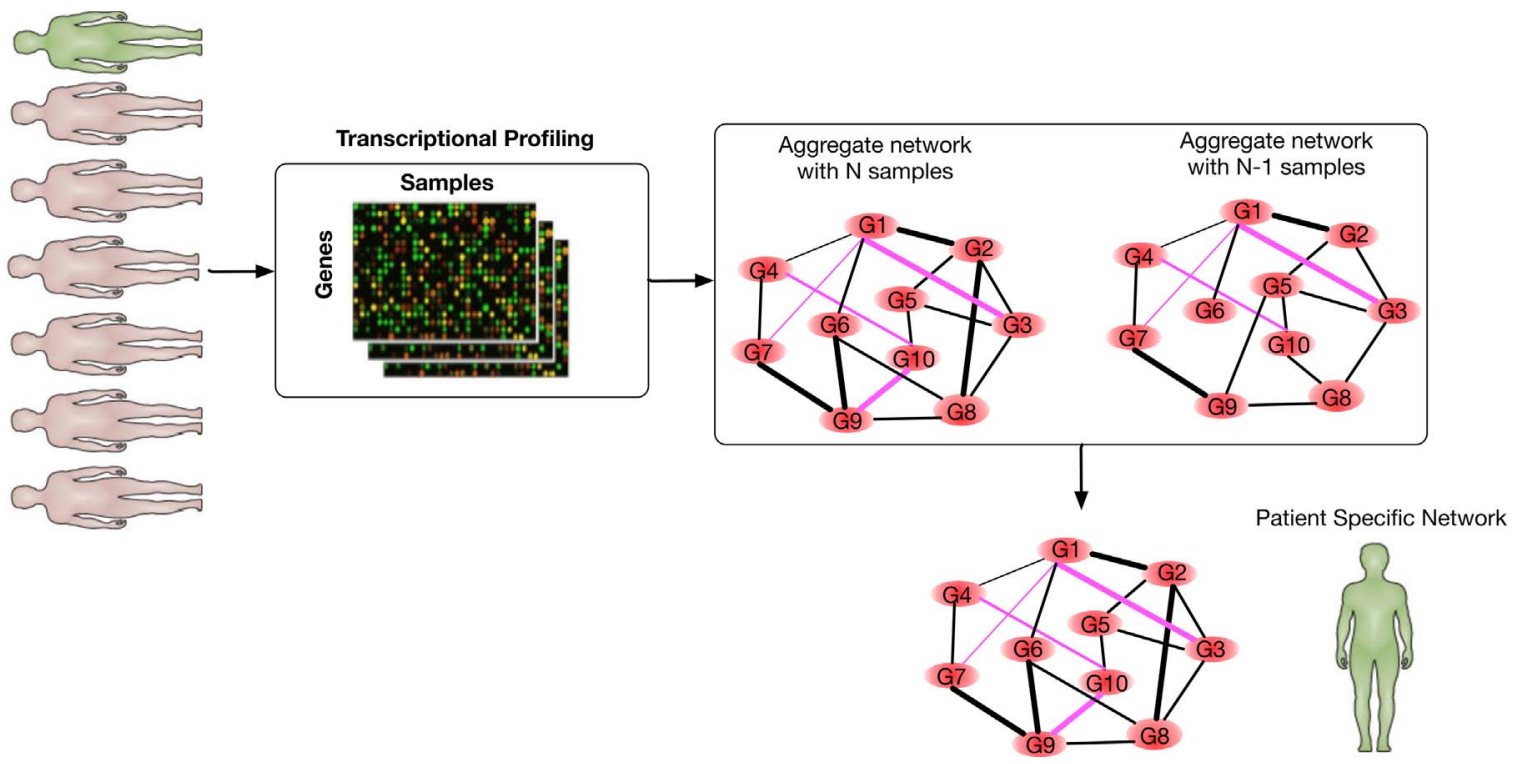

Fig. 7. Construction of patient specific network.

\section{Conclusions}

It is becoming evident that there are limits to personalized medicine as it is being practised today, generating high costs, and benefiting relatively few patients in proportion to the number of patient samples that are being assayed. Despite the tremendous importance of the genomic revolution being developed by various institutions, there is still a need to develop collaborative programs that recognise and handle the limitations seen today [186], as combined analysis of biomarkers at the DNA and transcriptomic level will probably be able to have more clinical utility than either marker alone [187].

Since genomic alterations vary in their cooperation between cancer types [188], since single cell analysis may reveal that mutations found at a population level may not be reflected at an individual level [189], since different models of clonal evolution exist within and between tumors [190]; [191], since it is becoming evident that drugs working in the metastatic setting not necessarily translate to the same benefits in the adjuvant setting, suggesting a different biology in both settings, since combining molecular targeted agents of highly effective singleagents is not necessarily better than either alone [192,193]; it is evident that a reductionist approach will ultimately not enable integration of the existing morphological features and molecular data obtained with high-throughput technologies at the bulk and/or single cell level.

Ultimately, this necessitates new models of data-analysis and incorporation of these models into new clinical trial designs with an appropriate data sharing policy [194]. As has been seen in complex trait analysis, the combined use of network science and reductionistic approaches will probably better capture the linear and nonlinear aspects of cancer biology [195].

A network science approach in cancer biology has been demonstrated to capture relevant targets that could not have been found with a reductionistic approach [196,197]. A network science approach is needed that will be able to integrate all relevant variables needed for a particular purpose, exemplified by the development of genome-scale model for drug target and biomarker identification [198,199,200,201,188,202].

It is imperative that the detection of multi-parametric biomarkers through network science approaches follows the same rigor in the development path of any biomarker and assay $[203,168]$ if they are to be included in a clinical trial context aimed at treating patients.

Only by applying rigorous patient-centered standards at all levels of knowledge of cancer biology, in the process of validation of biomarkers and in the consequent validation and implementation of new assays within a clinical trial and ultimately in a healthcare setting will we able to meet the promise of Precision Medicine at the individual patient level [204,205,206,207].

\section{Acknowledgements}

VSKM was supported by the Cancer Research Society (Canada). CS and RS were supported by the Breast Cancer Research Foundation (BCRF). CS was supported by FNRS (Fonds de la Recherche Scientifique) and PA and RS were supported by Federation Contre le Cancer. BHK was supported by the Gattuso-Slaight Personalized Cancer 

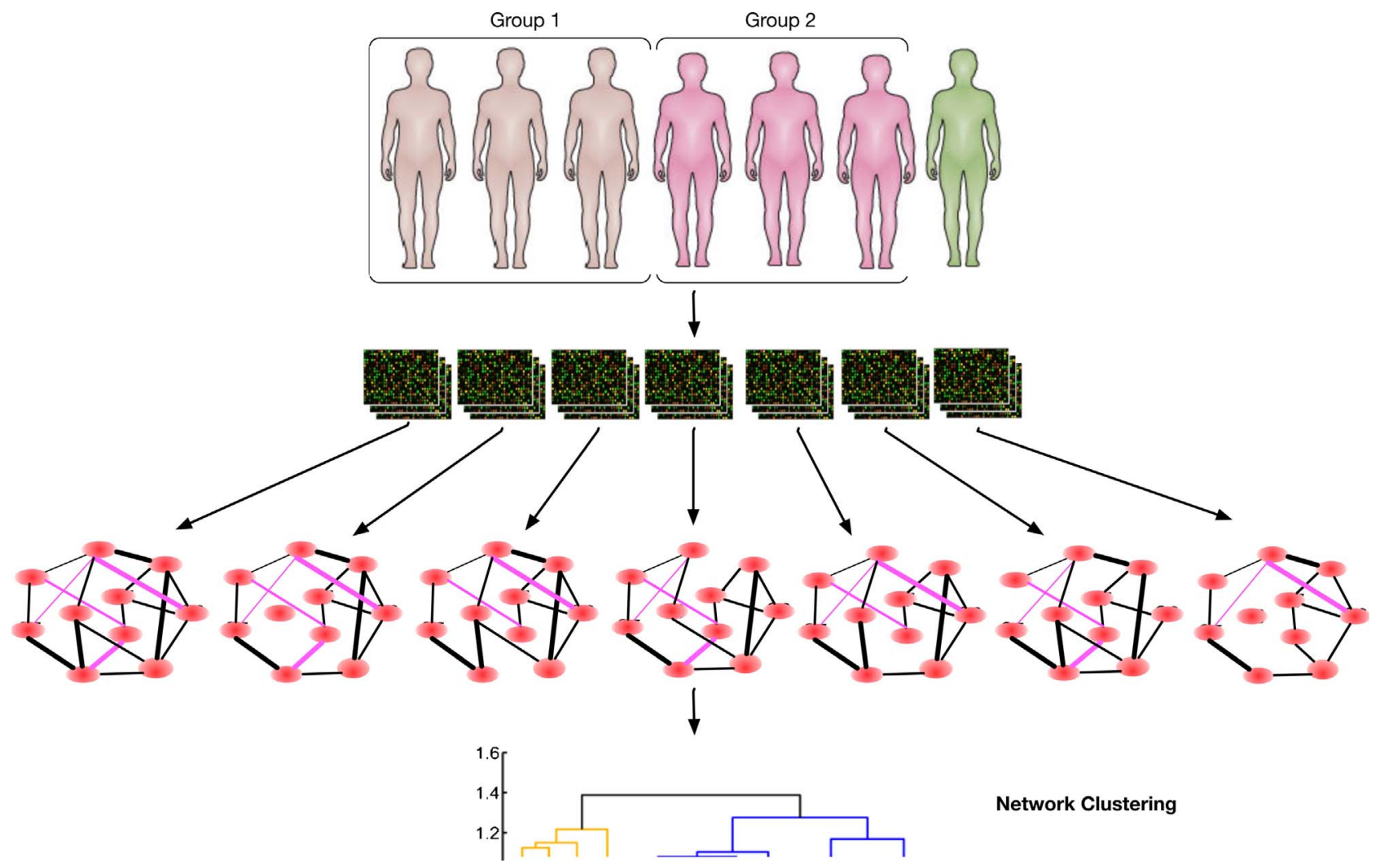

Network Clustering

Fig. 8. Clustering of patient specific networks.

Medicine Fund at Princess Margaret Cancer Centre, the Canadian Institutes of Health Research, and the Ontario Institute for Cancer Research through funding provided by the Government of Ontario.

\section{References}

[1] Zenggang Li, Andrei A. Ivanov, Rina Su, Valentina Gonzalez-Pecchi, Qi Qi, Songlin Liu, Philip Webber, et al., The OncoPPi network of cancer-focused proteinprotein interactions to inform biological insights and therapeutic strategies, Nat. Commun. 8 (February) (2017) 14356.

[2] Alice T. Shaw, Peggy P. Hsu, Mark M. Awad, Jeffrey A. Engelman, Tyrosine kinase gene rearrangements in epithelial malignancies, Nat. Rev. Cancer 13 (11) (2013) $772-787$.

[3] Levi A. Garraway, José Baselga, Whole-genome sequencing and cancer therapy: is too much ever enough? Cancer Discov. 2 (9) (2012) 766-768.

[4] Vessela N. Kristensen, Ole Christian Lingjærde, Hege G. Russnes, Hans Kristian M. Vollan, Arnoldo Frigessi, Anne-Lise Børresen-Dale, Principles and methods of integrative genomic analyses in cancer, Nat. Rev. Cancer 14 (5) (2014) 299-313.

[5] Walter Kolch, Melinda Halasz, Marina Granovskaya, Boris N. Kholodenko, The dynamic control of signal transduction networks in cancer cells, Nat. Rev. Cancer 15 (9) (2015) 515-527.

[6] Jeffery M. Klco, David H. Spencer, Christopher A. Miller, Malachi Griffith, Tamara L. Lamprecht, M. O'Laughlin, Catrina Fronick, et al., Functional heterogeneity of genetically defined subclones in acute myeloid leukemia, Cancer Cell 25 (3) (2014) 379-392.

[7] Jörg Menche, Amitabh Sharma, Maksim Kitsak, Susan Dina Ghiassian, Marc Vidal, Joseph Loscalzo, Albert-László Barabási, Disease networks. Uncovering diseasedisease relationships through the incomplete interactome, Science 347 (6224) (2015) 1257601.

[8] Brian A. Kidd, Ben P. Readhead, Caroline Eden, Samir Parekh, Joel T. Dudley, Integrative network modeling approaches to personalized cancer medicine, Pers. Med. 12 (3) (2015) 245-257.

[9] Matthew H. Spitzer, Yaron Carmi, Nathan E. Reticker-Flynn, Serena S. Kwek, Deepthi Madhireddy, Maria M. Martins, Pier Federico Federico Gherardini, et al., Systemic immunity is required for effective cancer immunotherapy, Cell 168 (3) (2017) 487-502 (e15).

[10] M.H. Spitzer, P.F. Gherardini, G.K. Fragiadakis, N. Bhattacharya, R.T. Yuan, A.N. Hotson, R. Finck, et al., An interactive reference framework for modeling a dynamic immune system, Science 349 (6244) (2015) (1259425-1259425).

[11] Daniel S. Chen, Ira Mellman, Elements of cancer immunity and the cancer-immune set point, Nature 541 (7637) (2017) 321-330.

[12] Natini Jinawath, Sacarin Bunbanjerdsuk, Maneerat Chayanupatkul, Nuttapong Ngamphaiboon, Nithi Asavapanumas, Jisnuson Svasti,
Varodom Charoensawan, Bridging the gap between clinicians and systems biologists: from network biology to translational biomedical research, J. Transl. Med. 14 (1) (2016) 324

[13] Jessica Xin Hu, Cecilia Engel Thomas, Søren Brunak, Network biology concepts in complex disease comorbidities, Nat. Rev. Genet. 17 (10) (2016) 615-29.

[14] Douglas Hanahan, Robert A. Weinberg, The hallmarks of cancer, Oxford Textbook of Oncology, (2015), pp. 3-10.

[15] Douglas Hanahan, Robert A. Weinberg, Hallmarks of cancer: the next generation, Cell 144 (5) (2011) 646-674.

[16] Lauren M.F. Merlo, John W. Pepper, Brian J. Reid, Carlo C. Maley, Cancer as an evolutionary and ecological process, Nat. Rev. Cancer 6 (12) (2006) 924-935.

[17] Franziska Michor, Yoh Iwasa, Martin A. Nowak, Dynamics of cancer progression, Nat. Rev. Cancer 4 (3) (2004) 197-205.

[18] Mel Greaves, Carlo C. Maley, Clonal evolution in cancer, Nature 481 (7381) (2012) 306-313.

[19] Ivana Bozic, Tibor Antal, Hisashi Ohtsuki, Hannah Carter, Dewey Kim, Sining Chen, Rachel Karchin, Kenneth W. Kinzler, Bert Vogelstein, Martin A. Nowak, Accumulation of driver and passenger mutations during tumor progression, Proc. Natl. Acad. Sci. U. S. A. 107 (43) (2010) 18545-18550.

[20] Shantanu Banerji, Kristian Cibulskis, Claudia Rangel-Escareno, Kristin K. Brown, Scott L. Carter, Abbie M. Frederick, Michael S. Lawrence, et al., Sequence analysis of mutations and translocations across Breast cancer subtypes, Nature 486 (7403) (2012) 405-409.

[21] Cancer Genome Atlas Research Network, Comprehensive genomic characterization defines human glioblastoma genes and core pathways, Nature 455 (7216) (2008) 1061-1068.

[22] Network, The Cancer Genome Atlas Research, Corrigendum: comprehensive genomic characterization defines human glioblastoma genes and core pathways, Nature 494 (7438) (2013) 506.

[23] Marcin Imielinski, Alice H. Berger, Peter S. Hammerman, Bryan Hernandez, Trevor J. Pugh, Eran Hodis, Jeonghee Cho, et al., Mapping the hallmarks of lung adenocarcinoma with massively parallel sequencing, Cell 150 (6) (2012) 1107-1120.

[24] Biankin, V. Andrew, Nicola Waddell, Karin S. Karin Kassahn, MarieClaude Gingras, Lakshmi B. Muthuswamy, Amber L. Johns, David K. Miller, et al., Pancreatic cancer genomes reveal aberrations in axon guidance pathway genes, Nature 491 (7424) (2012) 399-405.

[25] Cancer Genome Atlas Research Network, Integrated genomic analyses of ovarian carcinoma, Nature 474 (7353) (2011) 609-615.

[26] Network, Network, The Cancer Genome Atlas Research, and The Cancer Genome Atlas Research Network, Erratum: integrated genomic analyses of ovarian carcinoma, Nature 490 (7419) (2013) (292-292)

[27] P. Nowell, The clonal evolution of tumor cell populations, Science 194 (4260) (1976) 23-28.

[28] Eric R. Fearon, Bert Vogelstein, A genetic model for colorectal tumorigenesis, Cell 
61 (5) (1990) 759-767.

[29] B. Vogelstein, N. Papadopoulos, V.E. Velculescu, S. Zhou, L.A. Diaz, K.W. Kinzler, Cancer genome landscapes, Science 339 (6127) (2013) 1546-1558.

[30] Olga V. Razorenova, Amato J. Giaccia, Hypoxia, gene expression, and metastasis, The Tumor Microenvironment, (2010), pp. 43-58.

[31] Rob A. Cairns, Isaac S. Harris, Tak W. Mak, Regulation of cancer cell metabolism, Nat. Rev. Cancer 11 (2) (2011) 85-95.

[32] A Decade of Nature Cell Biology, Nat. Cell Biol. 11 (12) (2009) 1389-1390.

[33] D. Koschützki, F. Schreiber, Centrality analysis methods for biological networks and their application to gene regulatory networks, Gene Regul. Syst. Biol. 2 (May) (2008) 193-201.

[34] Olaf Sporns, Christopher J. Honey, Rolf Kötter, Identification and classification of hubs in brain networks, PLoS One 2 (10) (2007) e1049.

[35] Maksim Kitsak, Lazaros K. Gallos, Shlomo Havlin, Fredrik Liljeros, Lev Muchnik, H. Eugene Stanley, Hernán A. Makse, Identification of influential spreaders in complex networks, Nat. Phys. 6 (11) (2010) 888-893.

[36] Jian-Xiong Zhang, Duan-Bing Chen, Qiang Dong, Zhi-Dan Zhao, Identifying a set of influential spreaders in complex networks, Sci. Rep. 6 (2016) 27823.

[37] D. Koschützki, F. Schreiber, Centrality analysis methods for biological networks and their application to gene regulatory networks, Gene Regul. Syst. Biol. 2 (May) (2008) 193-201.

[38] Bill Andreopoulos, Aijun An, Xiaogang Wang, Michael Schroeder, A roadmap of clustering algorithms: finding a match for a biomedical application, Brief. Bioinf. 10 (3) (2009) 297-314.

[39] Megha Padi, John Quackenbush, Integrating transcriptional and protein interaction networks to prioritize condition-specific master regulators, BMC Syst. Biol. 9 (November) (2015) 80.

[40] H. Jeong, S.P. Mason, A.L. Barabási, Z.N. Oltvai, Lethality and centrality in protein networks, Nature 411 (6833) (2001) 41-42.

[41] Steve E. Calvano, Wenzhong Xiao, Daniel R. Richards, Ramon M. Felciano, Henry V. Baker, Raymond J. Cho, Richard O. Chen, et al., A network-based analysis of systemic inflammation in humans, Nature 437 (7061) (2005) 1032-1037.

[42] Sergei Maslov, Kim Sneppen, Specificity and stability in topology of protein networks, Science 296 (5569) (2002) 910-913.

[43] Albert-László Barabási, Natali Gulbahce, Joseph Loscalzo, Network medicine: a network-based approach to human disease, Nat. Rev. Genet. 12 (1) (2011) 56-68.

[44] John Platig, Peter J. Castaldi, Dawn DeMeo, John Quackenbush, Bipartite community structure of eQTLs, PLoS Comput. Biol. 12 (9) (2016) e1005033.

[45] M. Šikić, A. Lančić, N. Antulov-Fantulin, H. Štefančić, Epidemic centrality- is there an underestimated epidemic impact of network peripheral Nodes? Eur. Phys. J. B 86 (10) (2013), http://dx.doi.org/10.1140/epjb/e2013-31025-5.

[46] Tomasz Arodz, Danail Bonchev, Identifying influential nodes in a wound healingrelated network of biological processes using mean first-passage time, New J. Phys. 17 (2) (2015) 025002.

[47] David Gibbs, Ilya Shmulevich, Solving the Influence Maximization Problem Reveals Regulatory Organization of the Yeast Cell Cycle, (2016), http://dx.doi. org/10.1101/075069.

[48] Flaviano Morone, Hernán A. Makse, Corrigendum: influence maximization in complex networks through optimal percolation, Nature 527 (7579) (2015) 544.

[49] Ash A. Alizadeh, Victoria Aranda, Alberto Bardelli, Cedric Blanpain, Christoph Bock, Christine Borowski, Carlos Caldas, et al., Toward understanding and exploiting tumor heterogeneity, Nat. Med. 21 (8) (2015) 846-853.

[50] R. Rosenthal, N. han, J. Herrero, C. Swanton, Deciphering genetic intratumor heterogeneity and its impact on cancer evolution, Ann. Rev. Cancer Biol. 1 (1) (2016), http://dx.doi.org/10.1146/annurev-cancerbio-042516-011348.

[51] C. Swanton, Intratumor heterogeneity: evolution through space and time, Cancer Res. 72 (19) (2012) 4875-4882.

[52] M. Jamal-Hanjani, S.A. Quezada, J. Larkin, C. Swanton, Translational implications of tumor heterogeneity, Clin. Cancer Res. 21 (6) (2015) 1258-1266.

[53] Nicholas McGranahan, Charles Swanton, Biological and therapeutic impact of intratumor heterogeneity in cancer evolution, Cancer Cell 27 (1) (2015) 15-26.

[54] Marco Gerlinger, Andrew J. Rowan, Stuart Horswell, James Larkin, David Endesfelder, Eva Gronroos, Pierre Martinez, et al., Intratumor heterogeneity and branched evolution revealed by multiregion sequencing, N. Engl. J. Med. 366 (10) (2012) 883-892.

[55] Christopher R.S. Banerji, Simone Severini, Carlos Caldas, Andrew E. Teschendorff, Intra-tumour signalling entropy determines clinical outcome in Breast and lung cancer, PLoS Comput. Biol. 11 (3) (2015) e1004115.

[56] Park, Youngjune, S. Lim, J. Nam, S. Kim, Measuring intratumor heterogeneity by network entropy using RNA-Seq data, Sci. Rep. 6 (November) (2016) 37767.

[57] Douglas Hanahan, Lisa M. Coussens, Accessories to the crime: functions of cells recruited to the tumor microenvironment, Cancer Cell 21 (3) (2012) 309-322.

[58] Neil A. Bhowmick, Eric G. Neilson, Harold L. Moses, Stromal fibroblasts in cancer initiation and progression, Nature 432 (7015) (2004) 332-337.

[59] Johanna A. Joyce, Jeffrey W. Pollard, Microenvironmental regulation of metas tasis, Nat. Rev, Cancer 9 (4) (2009) 239-352.

[60] Margareta M. Mueller, Norbert E. Fusenig, Friends or foes - bipolar effects of the tumour stroma in cancer, Nat. Rev. Cancer 4 (11) (2004) 839-849.

[61] Greg Finak, Nicholas Bertos, Francois Pepin, Svetlana Sadekova, Margarita Souleimanova, Hong Zhao, Haiying Chen, et al., Stromal gene expression predicts clinical outcome in Breast cancer, Nat. Med. 14 (5) (2008) 518-527.

[62] E. Oh, S.M. Christensen, S. Ghanta, J. Jeong, O. Bucur, B. Glass, L. MontaserKouhsari, et al., Extensive rewiring of epithelial-stromal co-expression networks in breast cancer, Genome Biol. 16 (June) (2015) 128.

[63] Robert D. Schreiber, Lloyd J. Old, Mark J. Smyth, Cancer immunoediting: integrating immunity's roles in cancer suppression and promotion, Science 331 (6024)
(2011) 1565-1570.

[64] Peter Savas, Roberto Salgado, Carsten Denkert, Christos Sotiriou, Phillip K. Darcy, Mark J. Smyth, Sherene Loi, Clinical relevance of host immunity in Breast cancer: from TILs to the clinic, Clin. Oncol. 13 (4) (2016) 228-241.

[65] R. Salgado, C. Denkert, S. Demaria, N. Sirtaine, F. Klauschen, G. Pruneri, S. Wienert, et al., The evaluation of tumor-infiltrating lymphocytes (TILs) in breast cancer: recommendations by an international TILs working group 2014, Ann. Oncol. 26 (2) (2015) 259-271.

[66] Shuzhao Li, Helder I. Nakaya, Dmitri A. Kazmin, Jason Z. Oh, Bali Pulendran, Systems biological approaches to measure and understand vaccine immunity in humans, Semin. Immunol. 25 (3) (2013) 209-218.

[67] Ronald N. Germain, Martin Meier-Schellersheim, Aleksandra Nita-Lazar, Iain D.C. Fraser, Systems biology in immunology: a computational modeling perspective, Annu. Rev. Immunol. 29 (2011) 527-585.

[68] Damien Chaussabel, Nicole Baldwin, Democratizing systems immunology with modular transcriptional repertoire analyses, Nat. Rev. Immunol. 14 (4) (2014) 271-280.

[69] H. Tan, K. Yang, Y. Li, T. Shaw, Y. Wang, D. Blanco, X. Wang, et al., 2017. integrative proteomics and phosphoproteomics profiling reveals dynamic signaling networks and bioenergetics pathways underlying t cell activation, Immunity (2017), http://dx.doi.org/10.1016/j.immuni.2017.02.010.

[70] Alberto d' Onofrio, Metamodeling tumor-immune system interaction, tumor evasion and immunotherapy, Math. Comput. Modell. 47 (5-6) (2008) 614-637.

[71] Alberto d'Onofrio, A general framework for modeling tumor-Immune system competition and immunotherapy: mathematical analysis and biomedical inferences, Phys. D Nonlinear Phenom. 208 (3-4) (2005) 220-235.

[72] Arthur W. Lambert, Diwakar R. Pattabiraman, Robert A. Weinberg, Emerging biological principles of metastasis, Cell 168 (4) (2017) 670-691.

[73] Padmanee Sharma, Siwen Hu-Lieskovan, Jennifer A. Wargo, Antoni Ribas, Primary, adaptive, and acquired resistance to cancer immunotherapy, Cell 168 (4) (2017) 707-723.

[74] Ascierto, Maria Libera, Alvin Makohon-Moore, Evan J. Lipson, Janis M. Taube, Tracee L. McMiller, Alan E. Berger, Jinshui Fan, et al., Transcriptional mechanisms of resistance to anti-PD-1 therapy, Clin. Cancer Res. (2017) 17-0270, http://dx. doi.org/10.1158/1078-0432 (CCR-17-0270).

[75] Anagnostou, Valsamo, N. Kellie Smith, Patrick M. Forde, Noushin Niknafs, Rohit Bhattacharya, James White, T. Zhang, et al., Evolution of neoantigen landscape during immune checkpoint blockade in non-small cell lung cancer, Cancer Discov. 7 (3) (2016) 264-276.

[76] Teresa Davoli, Hajime Uno, Eric C. Wooten, Stephen J. Elledge, Tumor aneuploidy correlates with markers of immune evasion and with reduced response to immunotherapy, Science 355 (6322) (2017) eaaf8399.

[77] Gabriela Bindea, Bernhard Mlecnik, Marie Tosolini, Amos Kirilovsky, Maximilian Waldner, Anna C. Obenauf, Helen Angell, et al., Spatiotemporal dynamics of intratumoral immune cells reveal the immune landscape in human cancer, Immunity 39 (4) (2013) 782-795.

[78] Bernhard Mlecnik, Gabriela Bindea, Amos Kirilovsky, Helen K. Angell, Anna C. Obenauf, Marie Tosolini, Sarah E. Church, et al., The tumor microenvironmen and immunoscore are critical determinants of dissemination to distant metastasis, Sci. Transl. Med. 8 (327) (2016).

[79] Christian U. Blank, John B. Haanen, Antoni Ribas, Ton N. Schumacher, CANCER IMMUNOLOGY. the 'Cancer immunogram', Science 352 (6286) (2016) 658-660.

[80] Romain Remark, Taha Merghoub, Niels Grabe, Geert Litjens, Diane Damotte, Jedd D. Wolchok, Miriam Merad, Sacha Gnjatic, In-depth tissue profiling using multiplexed immunohistochemical consecutive staining on single slide, Sci. Immunol. 1 (1) (2016) aaf6925-aaf6925.

[81] Liu Rong, Cheng-Xian Guo, Hong-Hao Zhou, Network-based approach to identify prognostic biomarkers for estrogen Receptor-positive breast cancer treatment with tamoxifen, Cancer. Biol. Ther. 16 (2) (2015) 317-324.

[82] Andreas Heindl, Sidra Nawaz, Yinyin Yuan, Mapping spatial heterogeneity in the tumor microenvironment: a new era for digital pathology, Lab. Investig. J. Tech. Methods Pathol. 95 (4) (2015) 377-384.

[83] Patrik L. Ståhl, Fredrik Salmén, Sanja Vickovic, Anna Lundmark, José Fernández Navarro, Jens Magnusson, Stefania Giacomello, et al., Visualization and analysis of gene expression in tissue sections by spatial transcriptomics, Science 353 (6294) (2016) 78-82.

[84] Melissa R. Junttila, Frederic J. de Sauvage, Influence of tumour micro-environment heterogeneity on therapeutic response, Nature 501 (7467) (2013) 346-354.

[85] Feng Fu, Martin A. Nowak, Sebastian Bonhoeffer, Spatial heterogeneity in drug concentrations can facilitate the emergence of resistance to cancer therapy, PLoS Comput. Biol. 11 (3) (2015) e1004142.

[86] M. Mei-Chong Wendy, Fernando J. Lopez-Diaz, Shahid Yar Khan, Muhammad Akram Tariq, Yelena Dayn, Charles Joseph Vaske, Amie J. Radenbaugh, Hyunsung John Kim, Beverly M. Emerson, Nader Pourmand, Single-cell analyses of transcriptional heterogeneity during drug tolerance transition in cancer cells by RNA sequencing, Proc. Natl. Acad. Sci. U. S. A. 111 (44) (2014) E4726-E4735.

[87] D. Ramsköld, S. Luo, Y. Wang, R. Li, Q. Deng, O. Faridani, G.A. Daniels, et al., Fulllength mRNA-Seq from single-cell levels of RNA and individual circulating tumor cells, Nat. Biotechnol. 30 (8) (2012) 777-782.

[88] Megan Crow, Anirban Paul, Sara Ballouz, Z. Josh Huang, Jesse Gillis, Exploiting single-cell expression to characterize Co-expression replicability, Genome Biol. 17 (May) (2016) 101.

[89] Pau Creixell, Jüri Reimand, Syed Haider, Guanming Wu, Tatsuhiro Shibata, Miguel Vazquez, Ville Mustonen, et al., Pathway and network analysis of cancer genomes, Nat. Methods 12 (7) (2015) 615-621.

[90] Andrew L. Hopkins, Network pharmacology, Nat. Biotechnol. 25 (10) (2007) 
1110-1111.

[91] Albert Pujol, Roberto Mosca, Judith Farrés, Patrick Aloy, Unveiling the role of network and systems biology in drug discovery, Trends Pharmacol. Sci. 31 (3) (2010) 115-123.

[92] Shan Zhao, Ravi Iyengar, Systems pharmacology: network analysis to identify multiscale mechanisms of drug action, Annu. Rev. Pharmacol. Toxicol. 52 (2012) 505-521.

[93] Asfar S. Azmi, Zhiwei Wang, Philip A. Philip, Ramzi M. Mohammad, Fazlul H. Sarkar, Proof of concept: network and systems biology approaches aid in the discovery of potent anticancer drug combinations, Mol. Cancer Ther. 9 (12) (2010) 3137-3144.

[94] Tonekaboni Madani, Seyed Ali, Laleh Soltan Ghoraie, Venkata Satya Kumar Manem, Benjamin. Haibe-Kains, Predictive approaches for drug combination discovery in cancer, Briefings in Bioinformatics, (2016), http://dx.doi.org/10. 1093/bib/bbw104 November.

[95] Samira Jaeger, Ana Igea, Rodrigo Arroyo, Victor Alcalde, Begoña Canovas, Modesto Orozco, Angel R. Nebreda, Patrick Aloy, Quantification of pathway crosstalk reveals novel synergistic drug combinations for Breast cancer, Cancer Res. 77 (2) (2016) 459-469.

[96] Melinda Halasz, Boris N. Kholodenko, Walter Kolch, Tapesh Santra, Integrating network reconstruction with mechanistic modeling to predict cancer therapies, Sci. Signal. 9 (455) (2016) ra114.

[97] Igor Astsaturov, Vladimir Ratushny, Anna Sukhanova, Margret B. Finarson, Tetyana Bagnyukova, Yan Zhou, Karthik Devarajan, et al., Synthetic lethal screen of an EGFR-centered network to improve targeted therapies, Sci. Signal. 3 (140) (2010) ra67.

[98] Andrew P. Feinberg, Michael A. Koldobskiy, Anita Göndör, Epigenetic modulators, modifiers and mediators in cancer aetiology and progression, Nat. Rev. Genet. 17 (5) (2016) 284-299.

[99] Ian R. Watson, Koichi Takahashi, P. Andrew Futreal, Lynda Chin, Andrew futreal, and lynda chin. 2013. Emerging patterns of somatic mutations in cancer, Nat. Rev. Genet. 14 (10) (2013) 703-718

[100] Rodrigo Dienstmann, Louis Vermeulen, Justin Guinney, Scott Kopetz Sabine Tejpar, Josep Tabernero, Consensus molecular subtypes and the evolution of precision medicine in colorectal cancer, Nat. Rev. Cancer 17 (2) (2017) 79-92.

[101] Levi A. Garraway, Eric S. Lander, Lessons from the cancer genome, Cell 153 (1) (2013) 17-37.

[102] Steven A. Roberts, Dmitry A. Gordenin, Hypermutation in human cancer genomes: footprints and mechanisms, Nat. Rev. Cancer 14 (12) (2014) 786-800.

[103] Christoph Plass, Stefan M. Pfister, Anders M. Lindroth, Olga Bogatyrova, Rainer Claus, Peter Lichter, Mutations in regulators of the epigenome and their connections to global chromatin patterns in cancer, Nat. Rev. Genet. 14 (11) (2013) 765-780.

[104] Pau Creixell, Erwin M. Schoof, Janine T. Erler, Rune Linding, Navigating cance network attractors for tumor-specific therapy, Nat. Biotechnol. 30 (9) (2012) $842-848$.

[105] Osmanbeyoglu, U. Hatice, Eneda Toska, Carmen Chan, José Baselga, S. Leslie, Pancancer modelling predicts the context-specific impact of somatic mutations on transcriptional programs, Nat. Commun. 8 (January) (2017) 14249.

[106] Mark D.M. Leiserson, Fabio Vandin, Hsin-Ta Wu, Jason R. Dobson, Jonathan V. Eldridge, Jacob L. Thomas, Alexandra Papoutsaki, et al., Pan-cancer network analysis identifies combinations of rare somatic mutations across pathways and protein complexes, Nat. Genet. 47 (2) (2015) 106-114.

[107] James C. Costello, Laura M. Heiser, Elisabeth Georgii, Mehmet Gönen, Michael P. Menden, Nicholas J. Wang, Mukesh Bansal, et al., A community effort to assess and improve drug sensitivity prediction algorithms, Nat. Biotechnol. 32 (12) (2014) 1202-1212.

[108] Sreenath V. Sharma, Daniel A. Haber, Jeff Settleman, Line-based platforms to evaluate the therapeutic efficacy of candidate anticancer agents. nature reviews, Cancer 10 (4) (2010) 241-253.

[109] Pau Creixell, Antonio Palmeri, Chad J. Miller, Hua Jane Lou, Cristina C. Santini, Morten Nielsen, Benjamin E. Turk, Rune Linding, Unmasking determinants of specificity in the human kinome, Cell 163 (1) (2015) 187-201.

[110] Deepak L. Bhatt, Cyrus Mehta, Adaptive designs for clinical trials, N. Engl. J. Med. 375 (1) (2016) 65-74.

[111] Eunice L. Kwak, Yung-Jue Bang, D. Ross Camidge, Alice T. Shaw, Benjamin Solomon, Robert G. Maki, Sai-Hong I. Ou, et al., Anaplastic lymphoma kinase inhibition in non-small-cell lung cancer, N. Engl. J. Med. 363 (18) (2010) 1693-1703.

[112] Paul B. Chapman, Axel Hauschild, Caroline Robert, John B. Haanen, Paolo Ascierto, James Larkin, Reinhard Dummer, et al., Improved survival with vemurafenib in melanoma with BRAF V600E mutation, N. Engl. J. Med. 364 (26) (2011) 2507-2516.

[113] Tony S. Mok, Yi-Long Wu, Sumitra Thongprasert, Chih-Hsin Yang, Da-Tong Chu, Nagahiro Saijo, Patrapim Sunpaweravong, et al., Gefitinib or carboplatinPaclitaxel in pulmonary adenocarcinoma, N. Engl. J. Med. 361 (10) (2009) 947-957.

[114] Nicolas Stransky, Ethan Cerami, Stefanie Schalm, Joseph L. Kim, Christoph Lengauer, The landscape of kinase fusions in cancer, Nat. Commun. 5 (2014) 4846.

[115] Patients with NTRK Fusions Respond to Targeted Therapies, Cancer Discov. 6 (6) (2016) 566-567.

[116] Stefan Sleijfer, Jan Bogaerts, Lillian L. Siu, Designing transformative clinical trials in the cancer genome era, J. Clin. Oncol. 31 (15) (2013) 1834-41.

[117] Edward S. Kim, Roy S. Herbst, Ignacio I. Wistuba, J. Jack. Lee, R. George Blumenschein Jr, Anne Tsao, David J. David Stewart, et al., The BATTLE trial: personalizing therapy for lung cancer, Cancer Discov. 1 (1) (2011) 44-53.

[118] Fabrice André, Thomas Bachelot, Frederic Commo, Mario Campone, Monica Arnedos, Véronique Dieras, Magali Lacroix-Triki, et al., Comparative genomic hybridisation array and DNA sequencing to direct treatment of metastatic Breast cancer: a multicentre, prospective trial (SAFIR01/UNICANCER), Lancet Oncol. 15 (3) (2014) 267-274.

[119] Cancer Genome Atlas Network, Comprehensive molecular portraits of human Breast tumours, Nature 490 (7418) (2012) 61-70.

[120] Cancer Genome Atlas Research Network, Comprehensive molecular profiling of lung adenocarcinoma, Nature 511 (7511) (2014) 543-550.

[121] Justin Guinney, Rodrigo Dienstmann, Xin Wang, Andreas Schlicker Aurélien de Reyniès, Charlotte Soneson, Laetitia Marisa, et al., The consensus molecular subtypes of colorectal cancer, Nat. Med. 21 (11) (2015) 1350-1356.

[122] Cancer Genome Atlas Research Network, Cyriac Kandoth, Nikolaus Schultz, Andrew D. Cherniack, Rehan Akbani, Yuexin Liu, Hui Shen, et al., Integrated genomic characterization of endometrial carcinoma, Nature 497 (7447) (2013) $67-73$.

[123] Cancer Genome Atlas Research Network, Comprehensive molecular characterization of urothelial bladder carcinoma, Nature 507 (7492) (2014) 315-322.

[124] Cancer Genome Atlas Research Network, Comprehensive molecular characterization of gastric adenocarcinoma, Nature 513 (7517) (2014) 202-209.

[125] C. Kandoth, Michael D. McLellan, Fabio Vandin, Kai Ye, Beifang Niu, Charles Lu, Mingchao Xie, et al., Mutational landscape and significance across 12 major cancer types, Nature 502 (7471) (2013) 333-339.

[126] Bella Kaufman, Ronnie Shapira-Frommer, Rita K. Schmutzler, M. William Audeh, Michael Friedlander, Judith Balmaña, Gillian Mitchell, et al., Olaparib monotherapy in patients with advanced cancer and a germline BRCA1/2 mutation, J. Clin. Oncol. 33 (3) (2015) 244-250.

[127] David M. Hyman, Igor Puzanov, Vivek Subbiah, Jason E. Faris, Ian Chau, JeanYves Blay, Jürgen Wolf, et al., Vemurafenib in multiple nonmelanoma cancers with BRAF V600 mutations, N. Engl. J. Med. 373 (8) (2015) 726-736.

[128] Anirudh Prahallad, Chong Sun, Sidong Huang, Federica Di Nicolantonio, Ramon Salazar, Davide Zecchin, Roderick L. Beijersbergen, Alberto Bardelli, René Bernards, Unresponsiveness of colon cancer to BRAF(V600E) inhibition through feedback activation of EGFR, Nature 483 (7387) (2012) 100-103.

[129] Denis Lacombe, Susen Burock, Jan Bogaerts, Patrick Schoeffski, Vassilis Golfinopoulos, Roger Stupp, The dream and reality of histology agnostic cancer clinical trials, Mol. Oncol. 8 (6) (2014) 1057-1063.

[130] Travis Johnson, David. Liebner, James L. Chen, Opportunities for patient matching algorithms to improve patient care in oncology, JCO Clin. Cancer Inf. 1 (2017) $1-8$.

[131] Hope S. Rugo, Olufunmilayo I. Olopade, Angela DeMichele, Christina Yau, Laura J. van't Veer, Meredith B. Buxton, Michael Hogarth, et al., Adaptive randomization of veliparib-carboplatin treatment in Breast cancer, N. Engl. J. Med. 375 (1) (2016) 23-34.

[132] David Harrington, Giovanni Parmigiani, Adaptive randomization of neratinib in early breast cancer, N. Engl. J. Med. 375 (16) (2016) 1593-1594.

[133] Browning, Eiko Theodora, Andrew James James Weickhardt, D. Ross Camidge, Response to crizotinib rechallenge after initial progression and intervening chemotherapy in ALK lung cancer, J. Thorac. Oncol. 8 (3) (2013) e21.

[134] Tony S. Mok, Yi-Long Wu, Myung-Ju Ahn, Marina C. Garassino, Hye R. Kim, Suresh S. Ramalingam, Frances A. Shepherd, et al., Osimertinib or platinum-pemetrexed in EGFR T790M-positive lung cancer, N. Engl. J. Med. 376 (7) (2017) 629-640.

[135] AACR Project GENIE Consortium, AACR project GENIE: powering precision medicine through an international consortium, Cancer Discov. 7 (8) (2017) 818-831.

[136] Birgit Schoeberl, Art Kudla, Kristina Masson, Ashish Kalra, Michael Curley, Gregory Finn, Emily Pace, et al., Systems biology driving drug development: from design to the clinical testing of the anti-ErbB3 antibody seribantumab (MM-121), NPJ Syst. Biol. Appl. 3 (January) (2017) 16034.

[137] M.P. Smith, H. Brunton, E.J. Rowling, J. Ferguson, I. Arozarena, Z. Miskolczi, J.L. Lee, et al., Inhibiting drivers of non-mutational drug tolerance is a salvage strategy for targeted melanoma therapy, Cancer Cell 29 (3) (2016) 270-284.

[138] Fran Supek, Belén Miñana, Juan Valcárcel, Toni Gabaldón, Ben Lehner, Synonymous mutations frequently act as driver mutations in human cancers, Cell 156 (6) (2014) 1324-1335.

[139] Kalyana-Sundaram Shanker, Chandan Kumar-Sinha, Sunita Shankar, Dan R. Robinson, Yi-Mi Wu, Xuhong Cao, Irfan A. Asangani, et al., Expressed pseudogenes in the transcriptional landscape of human cancers, Cell 149 (7) (2012) $1622-1634$.

[140] S. Zhou, A.E. Treloar, M. Lupien, Emergence of the noncoding cancer genome: a target of genetic and epigenetic alterations, Cancer Discov. 6 (11) (2016) $1215-1229$.

[141] Julie George, Jing Shan Lim, Se Jin Jang, Yupeng Cun, Luka Ozretić, Gu Kong, Frauke Leenders, et al., Comprehensive genomic profiles of small cell lung cancer, Nature 524 (7563) (2015) 47-53.

[142] Yuan Yuan, Eliezer M. Van Allen, Larsson Omberg, Nikhil Wagle, Ali AminMansour, Artem Sokolov, Lauren A. Byers, et al., Assessing the clinical utility of cancer genomic and proteomic data across tumor types, Nat. Biotechnol. 32 (7) (2014) 644-652.

[143] Henrik Grönberg, Jan Adolfsson, Markus Aly, Tobias Nordström, Peter Wiklund, Yvonne Brandberg, James Thompson, et al., Prostate cancer screening in men aged 50-69 years (STHLM3): a prospective population-based diagnostic study, Lancet Oncol. 16 (16) (2015) 1667-1676.

[144] T. Pemovska, P. Ostling C. Heckman, O. Kallioniemi, Individualised systems 
medicine, Drugs (2015) (ddw-online.com), http://www.ddw-online.com/media/ 32/98212/individualised-systems-medicine.pdf.

[145] Michael J. Lee, Albert S. Ye, Alexandra K. Gardino, Anne Margriet Heijink, Peter K. Sorger, Gavin MacBeath, Michael B. Yaffe, Sequential application of anticancer drugs enhances cell death by rewiring apoptotic signaling networks, Cell 149 (4) (2012) 780-794.

[146] Lawrence N. Kwong, James C. Costello, Huiyun Liu, Shan Jiang, Timothy L. Helms, Aliete E. Langsdorf, David Jakubosky, et al., Oncogenic NRAS signaling differentially regulates survival and proliferation in melanoma, Nat. Med. 18 (10) (2012) 1503-1510.

[147] Lawrence N. Kwong, Timothy P. Heffernan, Lynda Chin, A systems biology approach to personalizing therapeutic combinations, Cancer Discov. 3 (12) (2013) 1339-1344.

[148] D.J. Slamon, B. Leyland-Jones, S. Shak, H. Fuchs, V. Paton, A. Bajamonde, T. Fleming, et al., Use of chemotherapy plus a monoclonal antibody against HER2 for metastatic breast cancer that overexpresses HER2, N. Engl. J. Med. 344 (11) (2001) 783-792.

[149] Martine J. Piccart-Gebhart, Marion Procter, Brian Leyland-Jones, Aron Goldhirsch, Michael Untch, Ian Smith, Luca Gianni, et al., Trastuzumab after adjuvant chemotherapy in HER2-Positive Breast cancer, N. Engl. J. Med. 353 (16) (2005) 1659-1672.

[150] D. Slamon, W. Eiermann, N. Robert, T. Pienkowski, M. Martin, M. Press, J. Mackey, et al., Adjuvant trastuzumab in HER2-Positive Breast cancer, N. Engl. J. Med. 365 (14) (2011) 1273-1283.

[151] A. Perez, Edward H. Romond, Vera J. Suman, Jong-Hyeon Jeong, George Sledge, Charles E. Geyer Jr, Silvana Martino, et al., Trastuzumab plus adjuvant chemotherapy for human epidermal growth factor receptor 2-positive breast cancer: planned joint analysis of overall survival from NSABP B-31 and NCCTG N9831, J. Clin. Oncol. 32 (33) (2014) 3744-3752.

[152] Charles E. Geyer, John Forster, Deborah Lindquist, Stephen Chan, C. Gilles Romieu, Tadeusz Pienkowski, Agnieszka Jagiello-Gruszfeld, et al., Lapatinib plus capecitabine for HER2-positive advanced breast cancer, N. Engl. J. Med. 355 (26) (2006) 2733-2743.

[153] Sandra M. Swain, José Baselga, Sung-Bae Kim, Jungsil Ro, Vladimir Semiglazov, Mario Campone, Eva Ciruelos, et al., Pertuzumab, trastuzumab, and docetaxel in HER2-positive metastatic Breast cancer, N. Engl. J. Med. 372 (8) (2015) 724-734.

[154] Sunil Verma, David Miles, Luca Gianni, Ian E. Krop, Manfred Welslau, José Baselga, Mark Pegram, et al., Trastuzumab emtansine for HER2-positive advanced breast cancer, N. Engl. J. Med. 367 (19) (2012) 1783-1791.

[155] Luca Gianni, Tadeusz Pienkowski, Young-Hyuck Im, Laslo Roman, LingMing Tseng, Mei-Ching Liu, Ana Lluch, et al., Efficacy and safety of neoadjuvant pertuzumab and trastuzumab in women with locally advanced, inflammatory, or early HER2-positive breast cancer (NeoSphere): a randomised multicentre, openlabel, phase 2 trial, Lancet Oncol. 13 (1) (2012) 25-32.

[156] José Baselga, Ian Bradbury, Holger Eidtmann, Serena Di Cosimo, Claudia Aura Evandro de Azambuja, Henry Gómez, et al., Lapatinib with trastuzumab for HER2-positive early breast cancer (NeoALTTO): a randomised, open-label, multicentre, phase 3 trial, Lancet 379 (9816) (2012) 633-640.

[157] Anthony Ferrari, Anne Vincent-Salomon, Xavier Pivot, Anne-Sophie Sertier, Emilie Thomas, Laurie Tonon, Sandrine Boyault, et al., A whole-genome sequence and transcriptome perspective on HER2-positive breast cancers, Nat. Commun. 7 (July) (2016) 12222.

[158] José Baselga, Javier Cortés, Seock-Ah Im, Emma Clark, Graham Ross, Astrid Kiermaier, Sandra M. Swain, Biomarker analyses in CLEOPATRA: a phase III, placebo-Controlled study of pertuzumab in human epidermal growth factor receptor 2-positive, first-line metastatic breast cancer, J. Clin. Oncol. 32 (33) (2014) 3753-3761.

[159] Sunil R. Lakhani, Marc J. Van De Vijver, Jocelyne Jacquemier, Thomas J. Anderson, Peter P. Osin, Lesley McGuffog, Douglas F. Easton, The pathology of familial Breast cancer: predictive value of immunohistochemical markers estrogen receptor, progesterone receptor, HER-2, and p53 in patients with mutations in BRCA1 and BRCA2, J. Clin. Oncol. 20 (9) (2002) 2310-2318.

[160] Amal Melhem-Bertrandt, Jasmina Bojadzieva, Kaylene J. Ready, Elias Obeid, Diane D. Liu, Angelica M. Gutierrez-Barrera, Jennifer K. Litton, et al., Early onset HER2-positive breast cancer is associated with germline TP53 mutations, Cancer 118 (4) (2012) 908-913.

[161] J.R.F. Wilson, A.C. Bateman, H. Hanson, Q. An, G. Evans, N. Rahman, J.L. Jones, D.M. Eccles, A novel HER2-positive breast cancer phenotype arising from germline TP53 mutations, J. Med. Genet, 47 (11) (2010) 771-774.

[162] Edith A. Perez, Sara A. Hurvitz, Lukas C. Amler, Kirsten E. Mundt, Vivian Ng, Ellie Guardino, Luca Gianni, Relationship between HER2 expression and efficacy with first-Line trastuzumab emtansine compared with trastuzumab plus docetaxel in TDM4450g: a randomized phase II study of patients with previously untreated HER2-Positive metastatic Breast cancer, Breast Cancer Res.: BCR 16 (3) (2014) R50.

[163] Maurizio Scaltriti, Paolo Nuciforo, Ian Bradbury, Jeff Sperinde, Dominique AgborTarh, Christine Campbell, Ahmed Chenna, et al., High HER2 expression correlates with response to the combination of lapatinib and trastuzumab, Clin. Cancer Res. 21 (3) (2015) 569-576.

[164] G. Gebhart, L.E. Lamberts, Z. Wimana, C. Garcia, P. Emonts, L. Ameye, S. Stroobants, et al., Molecular imaging as a tool to investigate heterogeneity of advanced HER2-positive breast cancer and to predict patient outcome under trastuzumab emtansine (T-DM1): the ZEPHIR trial, Anna. Oncol. 27 (4) (2016) 619-624.

[165] S. Loi, S. Michiels, R. Salgado, N. Sirtaine, V. Jose, D. Fumagalli, P.-L. KellokumpuLehtinen, et al., Tumor infiltrating lymphocytes are prognostic in triple negative breast cancer and predictive for trastuzumab benefit in early breast cancer: results from the FinHER trial, Ann. Oncol. 25 (8) (2014) 1544-1550.

[166] Stephen J. Luen, Roberto Salgado, Stephen Fox, Peter Savas, Jennifer Eng-Wong, Emma Clark, Astrid Kiermaier, et al., Tumour-infiltrating lymphocytes in advanced HER2-positive Breast cancer treated with pertuzumab or placebo in addition to trastuzumab and docetaxel: a retrospective analysis of the CLEOPATRA study, Lancet Oncol. 18 (1) (2017) 52-62.

[167] John Stagg, Sherene Loi, Upulie Divisekera, Shin Foong Ngiow, Helene Duret, Hideo Yagita, Michele W. Teng, Mark J. Smyth, Anti-ErbB-2 mAb therapy requires type I and II interferons and synergizes with anti-PD-1 or anti-CD137 mAb therapy, Proc. Natl. Acad. Sci. U. S. A. 108 (17) (2011) 7142-7147.

[168] Gary H. Lyman, Harold L. Moses, Biomarker tests for molecularly targeted therapies: laying the foundation and fulfilling the dream, J. Clin. Oncol. 34 (17) (2016) 2061-2066.

[169] Lisa M. McShanea, Margaret M. Cavenagh, Tracy G. Lively, David A. Eberhard, William L. Bigbee, P. Mickey Williams, Jill P. Mesirov, et al., Criteria for the use of omics-based predictors in clinical trials, Nature 502 (7471) (2013) 317-320.

[170] Beaver, A. Beulia, Abraham Tzou, Gideon M. Blumenthal, Amy E. McKee, Geoffrey Kim, Richard Pazdur, Reena Philip, An FDA perspective on the regulatory implications of complex signatures to predict response to targeted therapies, Clin. Cancer Res. 23 (March (6)) (2016) 1368-1372, http://dx.doi.org/10.1158/1078 0432. CCR-16-1098.

[171] Fred R. Hirsch, Paul A. Bunn Jr, Roy S. Herbst, 'Companion diagnostics': has their time come and gone? Clin. Cancer Res. 20 (17) (2014) 4422-4424.

[172] Steven J. Skates, A statistical challenge: developing tests for biomarker utility specific to the intended use, J. Natl. Cancer Inst. 106 (4) (2014) (dju076).

[173] S. Michiels, N. Ternès, F. Rotolo, Statistical controversies in clinical research: prognostic gene signatures are not (yet) useful in clinical practice, Ann. Oncol. 27 (12) (2016) 2160-2167.

[174] Chuang, Han-Yu, Eunjung Lee, Yu-Tsueng Liu, Doheon Lee, Trey Ideker, NetworkBased classification of Breast cancer metastasis, Mol. Syst. Biol. 3 (October) (2007) 140.

[175] Matan Hofree, John P. Shen, Hannah Carter, Andrew Gross, Trey Ideker, Networkbased stratification of tumor mutations, Nat. Methods 10 (11) (2013) 1108-1115.

[176] Felix K-H Chun, Pierre I. Karakiewicz, Alberto Briganti, Jochen Walz, Michael W. Kattan, Hartwig Huland, Markus Graefen, A critical appraisal of logistic regression-based nomograms, artificial neural networks, classification and regression-tree models, look-up tables and risk-group stratification models for prostate cancer, BJU Int. 99 (4) (2007) 794-800.

[177] Bo Wang, Aziz M. Mezlini, Feyyaz Demir, Marc Fiume, Zhuowen Tu, Michael Brudno, Benjamin Haibe-Kains, Anna Goldenberg, Similarity network fusion for aggregating data types on a genomic scale, Nat. Methods 11 (3) (2014) 333-337.

[178] Jeffrey S. Mogil, Malcolm R. Macleod, No publication without confirmation, Nature 542 (7642) (2017) 409-411.

[179] Marieke Lydia Kuijjer, Matthew Tung, Guocheng Yuan, John Quackenbush, Kimberly Glass, Estimating Sample-Specific Regulatory Networks, (2015) (arXiv [q-bio.MN]. arXiv), http://arxiv.org/abs/1505.06440.

[180] Morihiro Hayashida, Tatsuya Akutsu, Comparing biological networks via graph compression, BMC Syst. Biol. 4 (Suppl 2) (2010) S13 (September).

[181] Matthias Dehmer, Frank Emmert-Streib, Analysis of Microarray Data: A NetworkBased Approach, John Wiley \& Sons, 2008.

[182] Matthias Dehmer, Frank Emmert-Streib, Comparing large graphs efficiently by margins of feature vectors, Appl. Math. Comput. 188 (2) (2007) 1699-1710.

[183] Laurin A.J. Mueller, Karl G. Kugler, Armin Graber, Frank Emmert-Streib, Matthias Dehmer, Structural measures for network biology using QuACN, BMC Bioinf. 12 (December) (2011) 492.

[184] Beatriz Luna, Edgardo Galán-Vásquez, Edgardo Ugalde, Agustino MartínezAntonio, Structural comparison of biological networks based on dominant vertices, Mol. Biosyst. 9 (7) (2013) 1765-1773.

[185] Roded Sharan, Trey Ideker, Modeling cellular machinery through biological network comparison, Nat. Biotechnol. 24 (4) (2006) 427-433.

[186] Ian F. Tannock, John A. Hickman, Limits to precision cancer medicine, N. Engl. J. Med. 376 (1) (2017) 96-97.

[187] Emile E. Voest, Rene Bernards, DNA-guided precision medicine for cancer: a case of irrational exuberance? Cancer Discov. 6 (2) (2016) 130-132.

[188] Solip Park, Ben Lehner, Cancer type-dependent genetic interactions between cancer driver alterations indicate plasticity of epistasis across cell types, Mol. Syst. Biol. 11 (7) (2015) 824

[189] Xun Xu, Yong Hou, Xuyang Yin, Li Bao, Aifa Tang, Luting Song, Fuqiang Li, et al., Single-cell exome sequencing reveals single-nucleotide mutation characteristics of a kidney tumor, Cell 148 (5) (2012) 886-895.

[190] Baca, C. Sylvan, Davide Prandi, Michael S. Lawrence, Juan Mosquera, Alessandro Romanel, Yotam Drier, Kyung Park, et al., Punctuated evolution of prostate cancer genomes, Cell 153 (3) (2013) 666-677.

[191] Marc J. Williams, Benjamin Werner, Chris P. Barnes, Trevor A. Graham, Andrea Sottoriva, Identification of neutral tumor evolution across cancer types, Nat. Genet. 48 (3) (2016) 238-244.

[192] Raghav Sundar, Nicola Valeri, Kevin J. Harrington, A. Yap. Timothy, Combining molecularly targeted agents: is more always better? Clin. Cancer Res. 23 (5) (2017) 1123-1125.

[193] Bhupinder Mann, J. Zwiebel, Planning adjuvant trials when regimens effective for patients with advanced disease don't work in the adjuvant setting-paradigms lost, JAMA Oncol. (2017) s1, http://dx.doi.org/10.1001/jamaoncol.2017.0066.

[194] Robert L. Grossman, Allison P. Heath, Vincent Ferretti, Harold E. Varmus, Douglas R. Lowy, Warren A. Kibbe, Louis M. Staudt, Toward a shared vision for cancer 
genomic data, N. Engl. J. Med. 375 (12) (2016) 1109-1112.

[195] Evan G. Williams, Johan Auwerx, The convergence of systems and reductionist approaches in complex trait analysis, Cell 162 (1) (2015) 23-32.

[196] Nurcan Tuncbag, Pamela Milani, Jenny L. Pokorny, Hannah Johnson, Terence T. Sio, Simona Dalin, Dennis O. Iyekegbe, Forest M. White, Jann N. Sarkaria, Ernest Fraenkel, Network modeling identifies patient-specific pathways in glioblastoma, Sci. Rep. 6 (June) (2016) 28668.

[197] Tulin Ersahin, Nurcan Tuncbag, Rengul Cetin-Atalay, The PI3 K/AKT/mTOR in teractive pathway, Mol. Biosyst. 11 (7) (2015) 1946-1954.

[198] Adil Mardinoglu, Mathias Uhlen, Jens Nielsen, The use of genome-scale metabolic models for drug target and biomarker identification, New Biotechnol. 31 (2014) S49.

[199] Basti Bergdahl, Nikolaus Sonnenschein, Daniel Machado, Markus Herrgård, Jochen Förster, Genome-Scale models, Fundam. Bioeng. (2015) 143-182.

[200] Adam Friedman, Norbert Perrimon, Genetic screening for signal transduction in the era of network biology, Cell 128 (2) (2007) 225-231.

[201] Juanita S. Lopez, Udai Banerji, Combine and conquer: challenges for targeted therapy combinations in early phase trials, Nat. Rev. Clin. Oncol. 14 (1) (2016) $57-66$.

[202] Lee Hartwell, David Mankoff, Amanda Paulovich, Scott Ramsey,

Elizabeth Swisher, Cancer biomarkers: a systems approach, Nat. Biotechnol. 24 (8) (2006) 905-908.

[203] Daniel F. Hayes, Jeff Allen, Carolyn Compton, Gary Gustavsen, Debra G.B. Leonard, Robert McCormack, Lee Newcomer, et al., Breaking a vicious cycle, Sci. Transl. Med. 5 (196) (2013) $196 \mathrm{~cm} 6$.

[204] J. Jameson, J. Larry Jameson, Dan L. Longo, Precision medicine-personalized, problematic, and promising, N. Engl. J. Med. 372 (23) (2015) 2229-2234.

[205] H. West, Can we define and reach precise goals for precision medicine in cancer care? J. Clin. Oncol. (2016), http://dx.doi.org/10.1200/JCO.2016.68.8226.

[206] Hagop Kantarjian, Peter Paul Yu, Artificial intelligence, big data, and cancer JAMA Oncol. 1 (5) (2015) 573-574.

[207] I.S. Kohane, Ten things we have to do to achieve precision medicine, Science 349 (6243) (2015) 37-38. 